\title{
SIMULATION OF FREEFALL WATER ENTRY OF A FINITE WEDGE WITH FLOW DETACHMENT
}

\author{
C. M. $\mathrm{Bao}^{1}$, G. X. $\mathrm{Wu}^{* 1,2}$, G. $\mathrm{Xu}^{3}$ \\ 1. College of Shipbuilding Engineering, Harbin Engineering University, Harbin 150001, P. R. China; \\ 2. Department of Mechanical Engineering, University College London, London WC1E 7JE, UK; \\ 3. School of Naval Architecture and Ocean Engineering, Jiangsu university of science and technology, Zhenjiang \\ 212003, P. R. China;
}

\begin{abstract}
A two-dimensional finite wedge entering water obliquely in freefall with three degrees of freedom is considered through the velocity potential theory for the incompressible liquid. The problem is solved by using the boundary element method in the time domain. The scheme of the stretched coordinate system is adopted at the initial stages when only a small part of the wedge near its tip has entered water. The auxiliary function method is adopted to decouple the nonlinear mutual dependence between the body motions in three degrees of freedom and the fluid flow. When the liquid has detached from the knuckle of the wedge, the free jet is treated through the momentum equation. The developed method is verified through existing results for one degree of freedom in vertical motion. Various case studies are undertaken for a wedge entering water vertically, obliquely and with rotational angles. Results are provided the accelerations, velocities, pressure distribution and free surface deformation, and the physical implications are discussed.
\end{abstract}

\section{Keywords:}

Oblique water entry; Boundary element method; Flow detachment with free jet; Free fall motion; Multi degrees of freedom; Auxiliary function.

\section{Introduction}

Fluid/structure impact is a major concern for marine and coastal structures. A typical example is slamming of a ship. At large heave and pitch motion, its bow can emerge from water and then reenter water at high speed. When that happens, the ship may also have sway and roll motions. In such a case, each cross section of the ship enters water obliquely together with rotational velocity. A two dimensional wedge is commonly used in analysis. One reason is that the cross section of high speed craft is V-shaped. Another important reason is that understanding obtained from a wedge is highly relevant to many more general cases.

There has been extensive research on problems related to fluid/wedge impact, based on the incompressible velocity potential theory on the basis that the Mach number in such a case is relatively small and the period of impact is very short. Based on whether water entry is in freefall or in the prescribed motion, and whether the wedge is finite or infinite in width, the work can be broadly divided into four categories. In the first category, an infinite wedge enters water with prescribed velocity, either constant or varying. In the first category, an infinite wedge enters water with prescribed velocity, either constant or varying. Dobrovol'skaya [1] used the complex conformal mapping to consider a symmetric wedge vertically entry water with constant speed. She obtained a self-similar solution which satisfied the nonlinear free surface boundary condition. Zhao \& Faltinsen

*Corresponding author. Tel.: +44 207679 3870; fax: +44 2073880180.

E-mail address: g.wu@ucl.ac.uk (G.X.Wu) 
[2] considered the same problem using the boundary element method in the time domain. Adopting the integral hodograph method and using the velocity magnitude and direction as the variables, Semenov \& Iafrati [3] solved the problem of vertical water entry of an asymmetric wedge. Using the Cauchy theorem for the complex potential, Xu et al. [4] solved the problem of oblique entry of an asymmetric wedge.

In the second category, the wedge is finite in width. However the entry speed is still prescribed as either constant or varying. In such a case, the flow will detach from the knuckle of the wedge shortly after initial impact. The flow after detachment is no longer self similar even at constant speed and zero gravity. Zhao et al. [5] considered vertical water entry of a symmetric wedge. The velocity continuity condition was imposed at the knuckle after flow detachment. They also carried an experiment to verify their numerical results. Iafrati \& Battistin [6] investigated a symmetrical wedge vertically entering the calm water at constant speed. Tassin et al. [7] used an analytical model based on the Logvinovich model for a finite wedge. Bao et al. [8] simulated oblique water entry for an asymmetrical wedge. The gravity effect was included. The fluid was assumed to leave the knuckle tangentially after flow detachment. The thin jet flow was treated through momentum equation from which the solution could be obtained independently and directly. Semenov \& Wu [9] obtained the self-similar solution of an expanding wedge. When the ratio of the expansion speed to the entry speed is below a limit, flow detachment can occur.

In the third category, an infinite wedge enters water in free fall motion. Before the body touches water, its acceleration is equal to that due to gravity. As the body enters water, the acceleration changes due to hydrodynamic force. It becomes unknown and has to be found from the solution of the problem. The body motion and the fluid flow is therefore fully coupled. Wu et al. [10] considered a symmetric wedge vertically entering water in free fall motion. The nonlinear mutual dependence of the body motion and fluid flow was decoupled by the auxiliary function method [11]. Experiment was also undertaken and the acceleration from the simulation was in good agreement with the measured data at initial stage. While the wedge in the above work has only one degree of freedom, or the body is allowed to move only vertically downwards, Xu et al. [12] considered water entry of a wedge through free fall in three degrees of freedom. When rotation is included, the flow is no longer self similar even when the speed is constant as the ratio of the translational velocity and the angular velocity can be a length scale. Stability and accuracy of the numerical simulation is more important in three degrees of freedom, as numerical error could lead to motion instability.

In the fourth category, the wedge entering water through free fall motion is finite in width. Sun [13] simulated a wedge entering water vertically. The results were compared with the experimental data of Aarsnes [14]. More recently, Wang et al. [15] further studied this problem numerically and experimentally. However, it is still limited to one degree of freedom. The present work considers a finite wedge entering water through free fall motion in three degrees of freedom. Before flow detachment, the problem is similar to that considered by $\mathrm{Xu}$ et al. [12] for an infinite wedge. However, gravity effect in the free surface boundary condition and on the hydrodynamic force will 
be included here. Major differences occur after flow detachment. For an infinite wedge, its wetted surface keeps increasing and most part will be eventually above the rotational centre located at the centre of gravity. The rotational moment will change its sign during water entry, which may stop the body to rotate continuously in one direction, as it does during ship capsize. For a finite wedge the wetted surface will be constant after flow detachment. The moment may remain in the same direction and the body may continue to rotate in the same direction. Furthermore, after flow detachment, free jets may be formed [8]. Its accuracy should be ensured as the body motion can be very much affected by numerical error.

In the following sections, we shall first give the mathematical formulation and numerical procedure. Brief discussions will then be given about the boundary element method for solving the velocity potential and about the auxiliary function method to decouple the mutual dependence between the body motion and fluid flow. Before providing numerical results, convergence study is undertaken and comparison is made with available numerical and experimental data. Extensive case studies are then provided to show the behaviour of the body motion in three degrees of freedom, as well as the corresponding pressure distribution and free surface deformation.

\section{Mathematical model and numerical procedure}

\subsection{Governing equation and boundary conditions}

The two-dimensional oblique water entry problem of finite width with breadth $\tilde{B}$ at the top through free fall motion is considered here. The water density $\tilde{\rho}$, the vertical velocity $\tilde{V}$ at the moment of entry and the breadth $\widetilde{B}$ are used for the nondimensionalisation. Subsequently, the parameters without $\sim$ are nondimensional. The problem is sketched in Fig. 1. A Cartesian coordinate system $O-x y$ fixed in the space is defined, in which $x$-axis is along the undisturbed water surface and $y$-axis is vertically upwards. Heel angle $\theta$ in the figure is the angle between the symmetry line of the wedge and the $y$-axis. The wedge is asymmetric and has left and right deadrise angles $\gamma_{1}$ and $\gamma_{2}$, respectively, and the angle between its symmetry line and its face is $\gamma$. These angles form the following relationships:

$$
\gamma_{1}=\frac{\pi}{2}+\theta-\gamma, \gamma_{2}=\frac{\pi}{2}-\theta-\gamma
$$

At $t=0$, the tip of the wedge is touching the calm free surface. We set the origin of the system at the point. The rotating centre is located at the centre of the gravity $G$. The distance between $G$ and the tip of the wedge is $l$. The translational velocity of the wedge at point $G$ is $\boldsymbol{U}=U \boldsymbol{i}-V \boldsymbol{j}$, and the rotational velocity about $G$ is $\boldsymbol{\Omega}=\omega \boldsymbol{k}$, where $\boldsymbol{i}$ and $\boldsymbol{j}$ are the unit vectors in the $x$ and $y$ directions respectively, and $\boldsymbol{k}=\boldsymbol{i} \times \boldsymbol{j}$. Here the minus sign before $V$ means that it is positive when the body moves downwards. We notice that $V=1$ at $t=0$ based on the way in which the parameters are defined. 


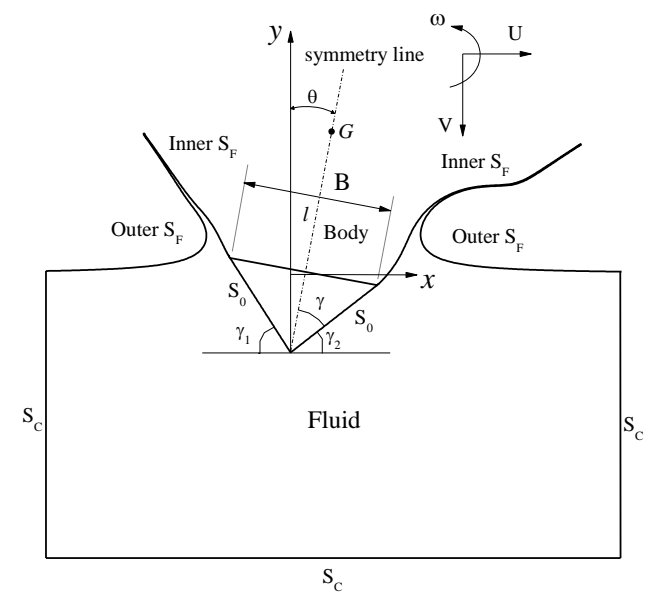

Fig. 1 Sketch of the problem

The fluid is assumed to be incompressible and inviscid, and the flow to be irrotational. A velocity potential $\phi$ can then be introduced, which satisfies Laplace equation

$$
\nabla^{2} \phi=0
$$

in the fluid domain. On the body surface $S_{0}$, we have from the impermeable condition

$$
\frac{\partial \phi}{\partial n}=(\boldsymbol{U}+\boldsymbol{\Omega} \times \boldsymbol{X}) \bullet \boldsymbol{n}=(U-\omega Y) n_{x}+(-V+\omega X) n_{y}
$$

where $\boldsymbol{n}=\left(n_{x}, n_{y}\right)$ is the normal vector of the body surface pointing out of the fluid domain. $\boldsymbol{X}=(X, Y)$ is the position vector relative to the centre of rotation. The Lagrangian form of the kinematic and dynamic conditions on the free surface $S_{F}$ can be written as

$$
\begin{aligned}
& \frac{D x}{D t}=\frac{\partial \phi}{\partial x}, \frac{D y}{D t}=\frac{\partial \phi}{\partial y} \\
& \frac{D \phi}{D t}=-\frac{y}{F r^{2}}+\frac{1}{2}|\nabla \phi|^{2}
\end{aligned}
$$

where $F r=\tilde{V} / \sqrt{\tilde{g} \tilde{B}}$ is the Froude number. The atmospheric pressure has been assumed to be constant in Eq. (5). In addition, we specify a far-field condition

$$
\frac{\partial \phi}{\partial n}=0
$$

on the basis that the fluid is undisturbed at $\sqrt{x^{2}+y^{2}} \rightarrow \infty$. A wedge impacting on water surface starts from a single point and the fluid domain noticeably disturbed by the wedge increases as the body moves into the water. To reflect that, we adopt the method of the stretched coordinate system [16]. We define

$$
\phi(x, y, t)=s(t) \varphi(\alpha, \beta, t), \alpha=x / s(t), \beta=y / s(t)
$$

where $s(t)$ is the vertical displacement of the centre of rotation:

$$
s(t)=\int_{0}^{t} V(\tau) d \tau
$$

Here it is expected that the vertical velocity $V$ is dominant in Eq. (8) during water entry. In the 
stretched coordinate system, the free surface boundary condition can be written as

$$
\begin{aligned}
& \frac{D(s \alpha)}{D t}=\frac{\partial \varphi}{\partial \alpha}, \frac{D(s \beta)}{D t}=\frac{\partial \varphi}{\partial \beta} \\
& \frac{D(s \varphi)}{D t}=-\frac{s \beta}{F r^{2}}+\frac{1}{2}\left(\varphi_{\alpha}^{2}+\varphi_{\beta}^{2}\right)
\end{aligned}
$$

The use of the stretched coordinate system is particularly effective when $s$ is small. When $s$ has reached a finite value, the simulation can continue in the stretched coordinate system or move back to the physical system $O-x y$.

\subsection{Numerical procedure}

Based on Green's second identity, the differential equation in the fluid domain can be converted into the following boundary integral equation.

$$
A(p) \varphi(p)=\int_{S}\left[\ln r_{p q} \frac{\partial \varphi(q)}{\partial n_{q}}-\varphi(q) \frac{\partial}{\partial n_{q}}\left(\ln r_{p q}\right)\right] d S_{q}
$$

where the whole boundary $S$ of the fluid domain includes the free surface $S_{\mathrm{F}}$, the body surface $S_{0}$ and the control surface $S_{\mathrm{C}}$ far away from the body. $A(p)$ is the solid angle of point $p$ on the body surface, and $r_{p q}$ is the distance between points $p$ and $q$.

The boundary of the fluid domain is discretized by using straight segments. On each element, the values of $\varphi$ and $\varphi_{n}$ are defined at the both nodes and their values within the element can be obtained by using the shape functions. We have

$$
f=N_{1}(\xi) f_{1}+N_{2}(\xi) f_{2}
$$

where $f$ stands for either $\varphi$ or $\varphi_{n}$, and

$$
N_{1}(\xi)=1-\xi, N_{2}(\xi)=\xi
$$

The local coordinate $\xi$ in the above equations varies from 0 at one node to 1 at the other. Eq. (11) can then be written as

$$
A(p) \varphi(p)+\sum_{k=1}^{N_{e}} \sum_{i=1}^{2} \varphi_{k}^{i}(q) \int_{0}^{1} \frac{\partial}{\partial n_{q}}\left(\ln r_{p q}\right) N_{i}(\xi) l_{k} d \xi=\sum_{k=1}^{N_{e}} \sum_{i=1}^{2} \frac{\partial \varphi_{k}^{i}(q)}{\partial n_{q}} \int_{0}^{1} \ln r_{p q} N_{i}(\xi) l_{k} d \xi
$$

where $i=1,2$ respectively denote the first and second nodes of the $k$ th element with length $l_{k}$ and $N_{e}$ is the total number of elements. By letting point $p$ approach each element node on the boundary, a system of equations can be obtained.

$$
[H]\{\varphi\}=[G]\left\{\varphi_{n}\right\}
$$

where the matrices $[H]$ and $[G]$ contain the integrals of $\partial\left(\ln r_{p q}\right) / \partial n_{q}$ and $\ln r_{p q}$ over each element [17], respectively, and $\{\varphi\}$ and $\left\{\varphi_{n}\right\}$ are columns containing the potentials and its normal derivatives on all the element nodes.

The normal derivative of the potential on the body surface and the potential on the free surface are known at each time step. They are moved to the right hand side of the equation, while the unknowns are moved to the left. Eq. (15) can then be rearranged as Lu et al. [17] 


$$
\left[\begin{array}{ll}
H^{S_{0}}-G^{S_{F}} & H^{S_{C}}
\end{array}\right]\left\{\begin{array}{l}
\varphi^{S_{0}} \\
\varphi_{n}^{S_{F}} \\
\varphi^{S_{C}}
\end{array}\right\}=\left[\begin{array}{lll}
G^{S_{0}}-H^{S_{F}} & G^{S_{C}}
\end{array}\right]\left\{\begin{array}{l}
\varphi_{n}^{S_{0}} \\
\varphi^{S_{F}} \\
\varphi_{n}^{S_{C}}
\end{array}\right\}
$$

where the superscripts correspond to the surfaces defined in Fig. 1. Once the solution of Eq. (16) at each time step has been obtained, we can update the free surface profile through the kinematic boundary conditions given by Eq. (4) while the dynamic boundary condition in Eq. (5) is used to update the velocity potential on the free surface.

When a wedge enters water, a jet can be usually observed, which requires some special treatments to avoid numerical error. Before flow detaches from the knuckle, the jet is attached to the body surface. The treatment used is similar to that used previously [16], in which the shallow water assumption is used. At the intersection of the body surface and the free surface, the velocity potential is continuous and is known at each time. However the normal derivative $\varphi_{n}$ is discontinuous. It is known when it is viewed from the body surface but unknown from the free surface, and can be obtained from the solution of Eq. (16). After the flow detaches from the knuckle, the jet becomes a free one with free surface on both sides. The motion of the free jet flow is governed by gravity. Following the method developed by Bao et al. [8], momentum equation for the jet can be written as

$$
\frac{D \boldsymbol{u}}{D t}=-\frac{1}{F r^{2}} \boldsymbol{j}
$$

The velocity in the jet flow region can be obtained from Eq. (17), and subsequently the position and potential of the jet surface can be obtained from Eqs. (4) and (5), respectively. As a result all the information on the jet surface required in Eq.(11) become known. At the intersection of the free surface and the body surface, we assume that the flow leaves the knuckle tangentially, $\varphi_{n}$ is therefore continuous at the knuckle and is known from the body surface boundary condition. The velocity potential at the intersection after detachment can be treated as unknown and can be obtained by solving Eqs. (16).

\subsection{The body motion}

When the potential has been found, the Bernoulli equation can be used to obtain the pressure:

$$
p=-\left(\phi_{t}+\frac{1}{2} \nabla \phi \nabla \phi+\frac{y}{F r^{2}}\right)
$$

in which the density of the fluid is absent after nondimensionalisation. The force $\boldsymbol{F}$ and the moment $\boldsymbol{M}$ acting on the body can be obtained by integrating the pressure over its wetted surface $S_{0}$ :

$$
\begin{gathered}
\boldsymbol{F}=\int_{S_{0}} p \boldsymbol{n} d S \\
\boldsymbol{M}=\int_{S_{0}} p(\boldsymbol{X} \times \boldsymbol{n}) d S
\end{gathered}
$$

Based on Newton's law, the equation for the body motion can be written as 


$$
\left[M_{b}\right][\dot{U}]=\left[F_{h}\right]+\left[F_{e}\right]
$$

where $\left[M_{b}\right]$ is the body mass matrix, $[\dot{U}]$ is a column of body acceleration with two translations and one rotation, $\left[F_{h}\right]$ is a column of the hydrodynamic force and moment, and $\left[F_{e}\right]$ is a column of the external force and moment such as the force due to the gravity.

By solving Eq. (11), $\phi$ can be found. However, $\phi_{t}$ is still not explicitly known. Although it could be obtained by a difference method with respect to time from results at different time steps, such a procedure is usually not accurate enough and sometimes may lead to numerical stability, subsequently the body motion. Here we adopt the approach proposed by Wu \& Eatock Taylor[11]. $\phi_{t}$ satisfies the Laplace equation in the fluid domain. Its free surface boundary condition can be obtained by letting Eq. (18) be equal to zero

$$
\phi_{t}=-\frac{1}{2} \nabla \phi \nabla \phi-\frac{y}{F r^{2}}
$$

The body surface boundary condition can be written as [18]:

$$
\frac{\partial \phi_{t}}{\partial n}=(\dot{\boldsymbol{U}}+\dot{\boldsymbol{\Omega}} \times \boldsymbol{X}) \cdot \boldsymbol{n}-\boldsymbol{U} \cdot \frac{\partial \nabla \phi}{\partial n}+\boldsymbol{\Omega} \cdot \frac{\partial}{\partial n}[\boldsymbol{X} \times(\boldsymbol{U}-\boldsymbol{\nabla} \phi)]
$$

From Eq. (23), the acceleration is needed for solving $\phi_{t}$. On the other side, the acceleration in Eq.(23) depends on $\phi_{t}$. Here we adopt the method developed by Wu \& Eatock Taylor [11] to decouple the nonlinear mutual dependence between the body motion and the hydrodynamic force.

We introduce some auxiliary functions $\chi$ and $\lambda_{i}(i=1,2,3)$ which satisfy Laplace equation, and write $\phi_{t}$ as

$$
\left.\phi_{t}=\chi-\boldsymbol{U} \cdot \nabla \phi+\Omega \cdot[\boldsymbol{X} \times(\boldsymbol{U}-\nabla \phi)]\right)+\dot{U}_{1} \lambda_{1}+\dot{U}_{2} \lambda_{2}+\dot{U}_{3} \lambda_{3}
$$

The boundary conditions for $\chi$ and $\lambda_{i}$ can be given based on those for $\phi_{t}$. On the body surface

$$
\begin{aligned}
& \frac{\partial \chi}{\partial n}=0 \\
& \frac{\partial \lambda_{i}}{\partial n}=n_{i}
\end{aligned}
$$

On the free surface

$$
\begin{gathered}
\chi=\boldsymbol{U} \cdot \nabla \phi-\boldsymbol{\Omega} \cdot[\boldsymbol{X} \times(\boldsymbol{U}-\nabla \phi)]-\frac{1}{2} \nabla \phi \nabla \phi-\frac{y}{F r^{2}} \\
\lambda_{i}=0
\end{gathered}
$$

On the control surface, the normal derivative of those auxiliary functions are zero. All the boundary conditions on $\chi$ and $\lambda_{i}(i=1,2,3)$ are known once the potential is found and those can be solved in a manner similar to that used for $\phi$. Using $\chi$ and $\lambda_{i}(i=1,2,3)$ into Eq. (21), we have

$$
\sum_{i=1}^{3}\left(\delta_{j i} M_{i i}+N_{j i}\right) \cdot \dot{U}_{i}=f_{j}-\frac{M_{i i} \delta_{j 2}}{F r^{2}}
$$

where 


$$
\begin{gathered}
\delta_{j i}=\left\{\begin{array}{ll}
1 & j=i \\
0 & j \neq i
\end{array}, \quad N_{j i}=\iint_{s_{0}} \lambda_{j} \bullet n_{i} d S\right. \\
\left.f_{j}=-\iint_{s_{0}}(\chi-\boldsymbol{U} \cdot \nabla \phi+\boldsymbol{\Omega} \cdot[\boldsymbol{X} \times(\boldsymbol{U}-\nabla \phi)])+\frac{1}{2} \nabla \phi \nabla \phi+\frac{y}{F r^{2}}\right) n_{j} d S
\end{gathered}
$$

and $\left(n_{1}, n_{2}, n_{3}\right)=\left(n_{x}, n_{y}, X n_{y}-Y n_{x}\right),\left(\dot{U}_{1}, \dot{U}_{2}, \dot{U}_{3}\right)=(\dot{U},-\dot{V}, \dot{\omega})$.

The body acceleration then can be obtained from Eq. (29), and the force $\boldsymbol{F}$ and the moment $\boldsymbol{M}$ can be found from Eq. (19) and Eq. (20), respectively.

\section{Numerical results and discussions}

We start the simulation at $s=s_{0}$ which can be chosen arbitrarily small. At such a small distance, the overall disturbance to the liquid is small. The initial solution can be chosen for the computational convenience as the result does not have large effect on the flow at later stage. Thus it can be selected to ensure that the simulation can move forward smoothly with the time step. Here we adopt the similarity solution as the initial solution at $s_{0}$, as done by $\mathrm{Xu}$ et al. [4], and use the time stepping method onward. In the present paper, we start our simulations at $s_{0}=0.001$. The computational domain is chosen as a rectangular control box which is truncated at $\alpha= \pm \max \left(20,20 / \tan \gamma_{i}\right)$ and $\beta=-40$ in the stretched coordinate system.

Elements of typical length $\Delta l$ are uniformly distributed on the body surface $S_{0}$. Before flow detachment, equal elements are used on the free surface near the body. On the free surface away from the body, the size of the element increases gradually at a fixed ratio, and the largest element far away from the body is about five times typical length $\Delta l$. After the flow detaches from the knuckle of the body, a local mesh refinement is used near the tip of the jet and the knuckle.

The time $\Delta t$ is chosen to ensure sufficient accuracy which is determined by

$$
\Delta t=C \frac{\min (\Delta l)_{\mathrm{SF}}}{\max \left(\mid(\nabla \phi)_{\mathrm{SF}}\right)}
$$

where $0<C<1$ is to ensure that the fluid particle on the free surface will move a small fraction of grid size within one time step. During the simulation, the time step will be adjusted based on the Eq.(31) through the ratio of the smallest element size and the largest velocity magnitude. When the free surface deforms as time increases, elements can be distorted severely and remeshing is therefore applied regularly based on the scheme used by Sun[13].

During water entry, the heel angle $\theta$ can be obtained by

$$
\theta(t)=\theta_{0}-\int_{0}^{t} \omega(\tau) d \tau
$$

Here and in other parts, the subscript 0 indicates the initial value. The horizontal displacement of the centre of rotation 


$$
h(t)=\int_{0}^{t} U(\tau) d \tau
$$

The horizontal and vertical displacements of the tip of the wedge are respectively

$$
s_{P}=s(t)+l\left(\cos \theta-\cos \theta_{0}\right)
$$

and

$$
h_{P}=h(t)+l\left(\sin \theta-\sin \theta_{0}\right)
$$

where $l$ is the distance between the tip and centre of the gravity of the wedge.

\subsection{Convergence study and comparison}

A wedge entering water vertically in free fall with flow detachment is simulated for convergence study. We choose the case of $\gamma_{1}=\gamma_{2}=30, F r=1.9587$ and $m=1.1722$, which is same as that in the experiment by Wang et al. [15]. We set the typical element length $\Delta l=0.04,0.03$ and 0.02 respectively in the stretched system. Fig. 2 gives the free surface profile and the pressure distribution after flow detachment at $s=0.4$, and the vertical acceleration. The results corresponding to different meshes are in a good agreement. This shows that the present method is already mesh independent. To verify convergence with the time step, we choose $C=0.4,0.3$ and 0.2 respectively. Fig. 3 gives results at $s=0.4$ and it can be seen that convergence has been achieved. $C$ is taken as 0.3 in the following simulations together with $\Delta l=0.03$.
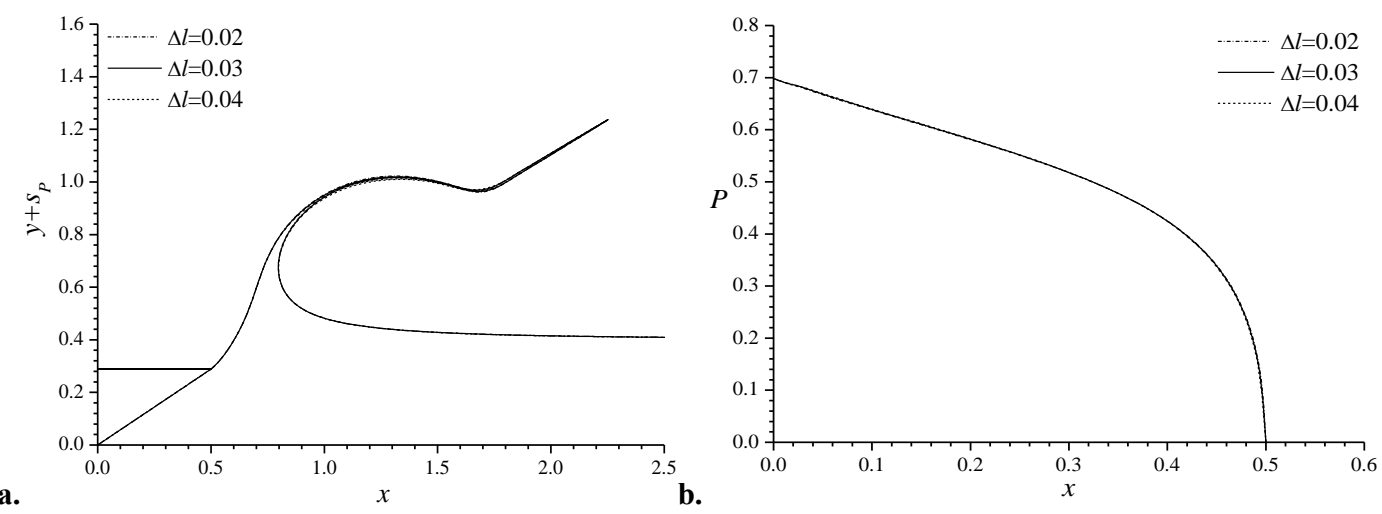

a.

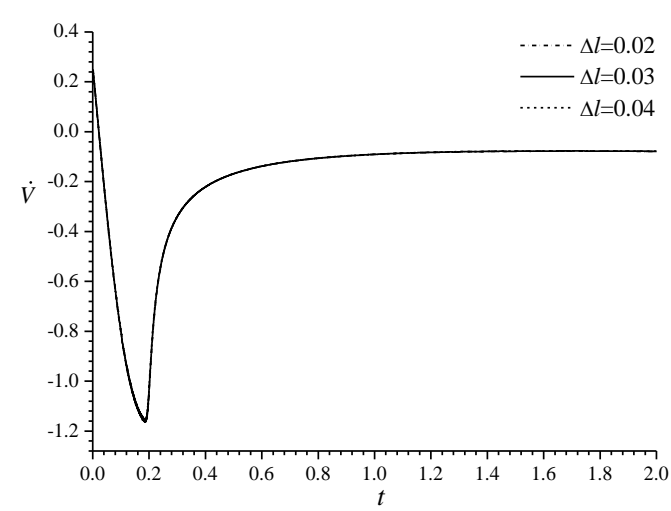

Fig. 2 Mesh convergence study, (a) free surface profile ( $s=0.4)$, (b) total pressure distribution on the wedge surface ( $s=0.4$ ), (c) vertical acceleration of a wedge with $\gamma_{1}=\gamma_{2}=30, F r=1.9587$ and $m=1.1722$ 

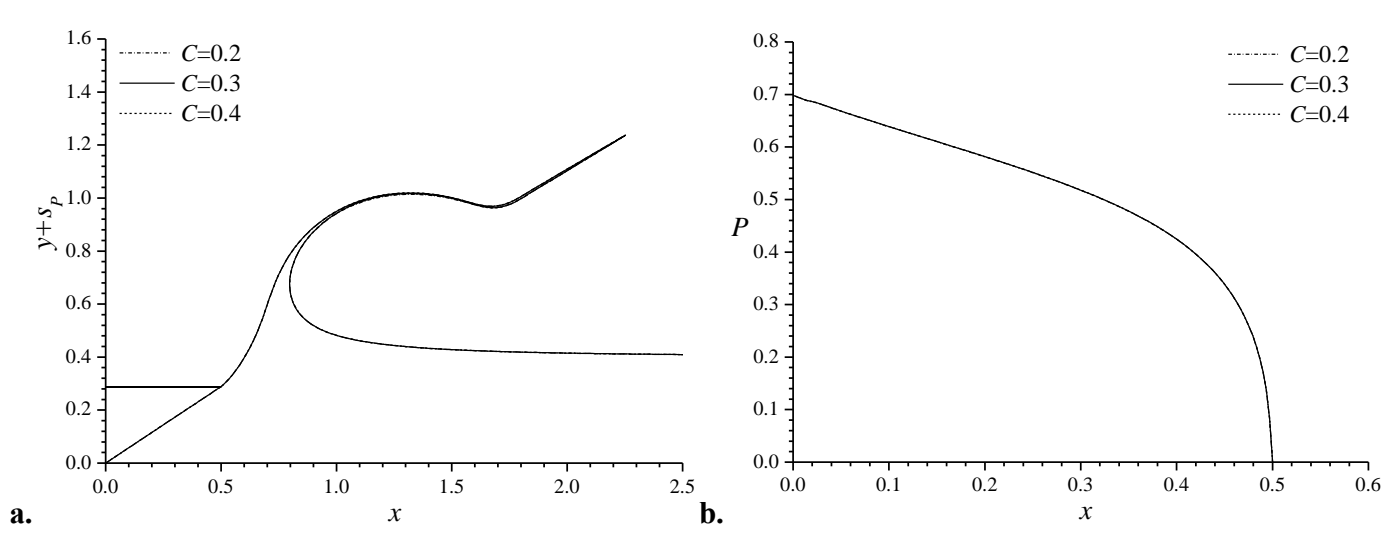

a.

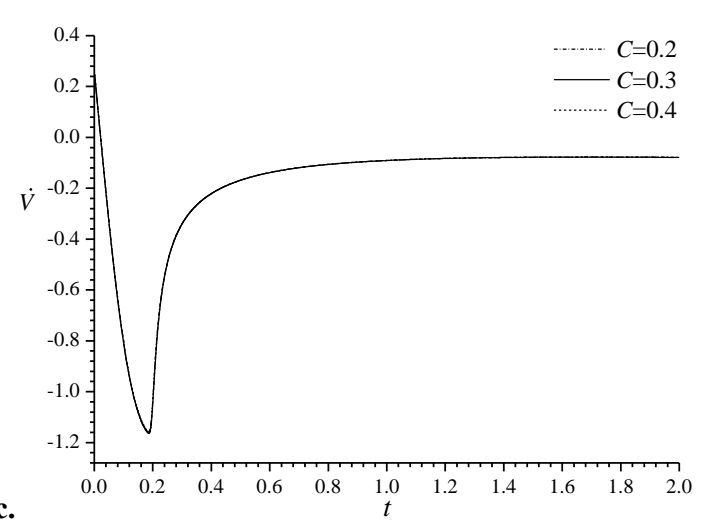

Fig. 3 Time step convergence study, (a) free surface profile $(s=0.4)$, (b) total pressure distribution on the wedge surface $(s=0.4)$, (c) vertical acceleration of a wedge with $\gamma_{1}=\gamma_{2}=30, F r=1.9587$ and $m=1.1722$

Then we verify our method by comparing with the results in Wang et al. [15], as shown in Fig.4. The vertical acceleration from the present numerical simulation agree very well with those obtained numerically by Wang et al. [15] in Fig. 4a. The experimental results by Wang et al. [15] are also provided. Although the trends are very similar, there is some noticeable difference between the numerical results and experiment data. It has been stated by Wang et al. [15] themselves that "when we have peak acceleration, the numerical pressures on the impact side agree well with experiments, which should result in a good agreement of the hydrodynamic force (and the body acceleration) between the numerical results and experiments. This causes a contradiction that we cannot explain." The task of offering a valid explanation here is obviously more difficult. Fig. 4 a further provides the acceleration for the wedge with the same parameters but much larger length, in which no flow detachment occurs. Before flow detachment, all the numerical results are virtually the same. At later stage, the attached flow obviously has a larger upward force and therefore the wedge has a larger upward acceleration, as shown in Fig. 4a and reflected by the velocity in Fig. 4b. Comparison for pressures from the present result and the numerical and experimental data of Wang et al. [15] is shown in Fig. 4c, where point P5 is on the body surface, and has a horizontal distance of 0.1145 to the tip of the wedge. Comparison with Wang et al. [15] for the free surface profiles at $t=0.7545$ and $t=2.6220$ is displayed in Fig. 5 and very good agreement can be found. 

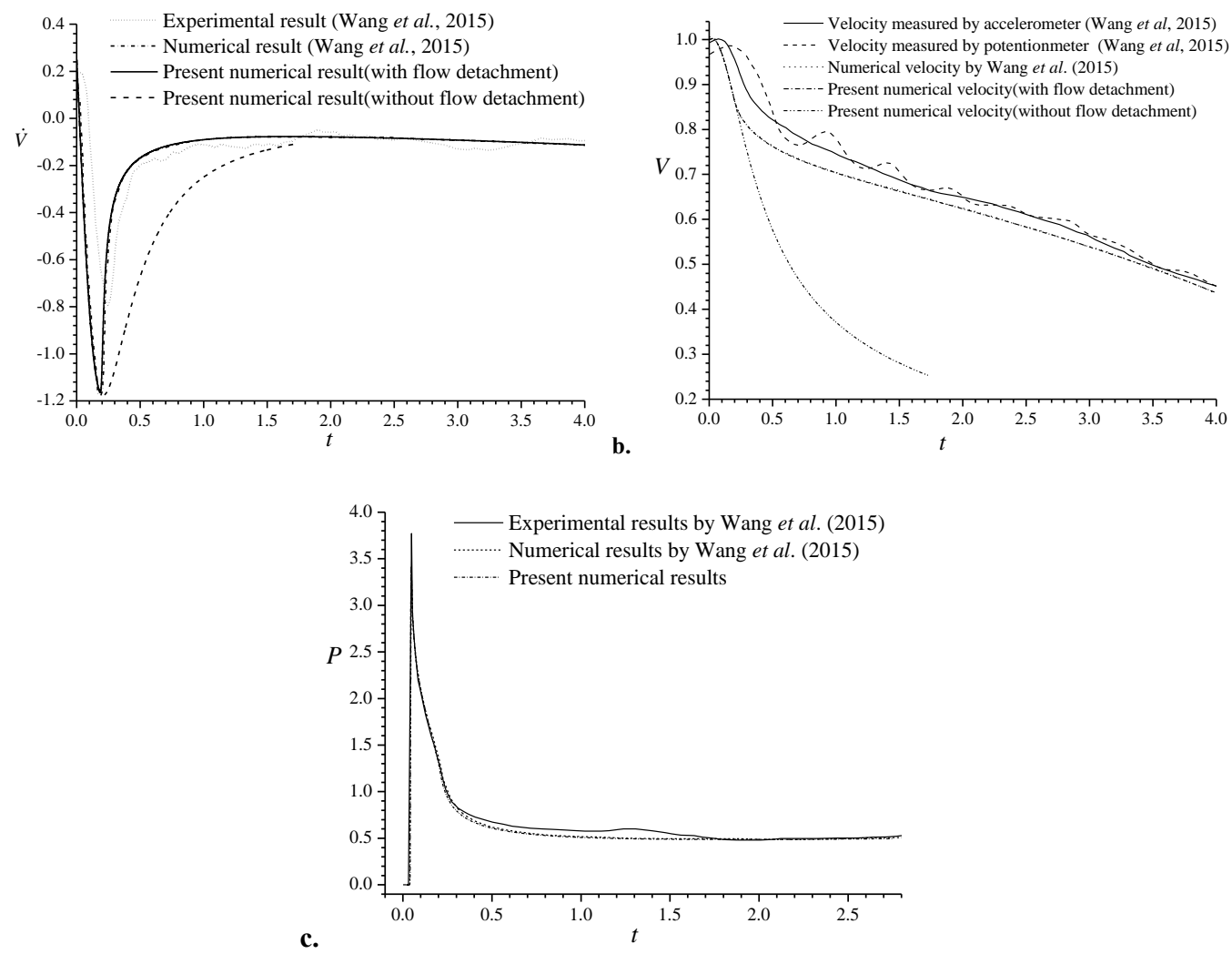

Fig. 4 Results for vertical water entry of a symmetric wedge with $\left(\gamma_{1}=\gamma_{2}=30^{\circ}, F r=1.9587, m=1.1722\right)$,

(a) acceleration, (b) vertical velocity, (c) press at point P5.
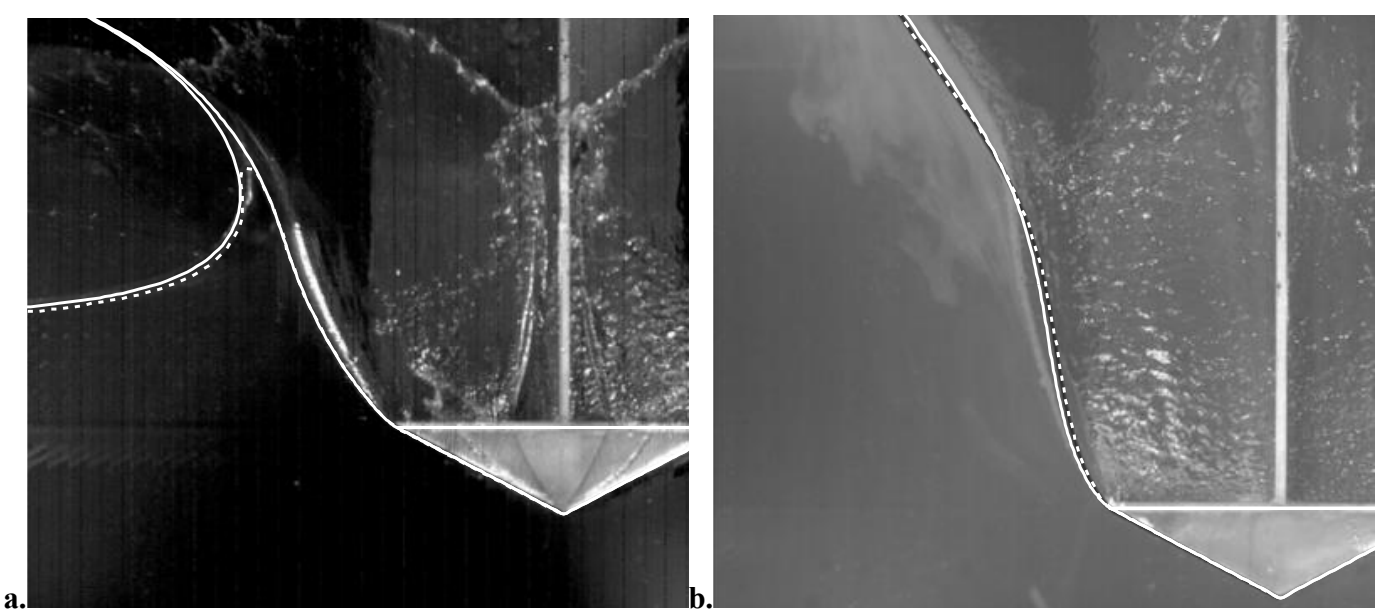

Fig. 5 Comparison of surface profiles between experimental results by Wang et al. [15] and numerical result for wedge $\left(\gamma_{1}=\gamma_{2}=30^{\circ}, F r=1.9587, m=1.1722\right)$ at (a) $t=0.7545$ and, (b) $t=2.6220$. short dash line, numerical results by Wang et al. [15]; solid line, the present numerical results

We further validate our numerical method by considering an asymmetric case. Drop tests from different heights were carried by Xu et al.[19], with initially zero or non-zero heel angle. Oger et al. [20] simulated one of the cases in Xu et al.[19] based on the smoothed particles hydrodynamics (SPH) method. The comparisons of vertical and rotational accelerations are respectively provided in Figs. 6a and 6b. The deadrise angles are much smaller than that in Fig.4 and therefore it is a computationally and experimentally more challenging case. Both results from Xu et al.[19] through 
model testing and from Oger et al. [20] through computational are quite oscillatory. The present results are in fairly good agreement with the mean lines of their results.
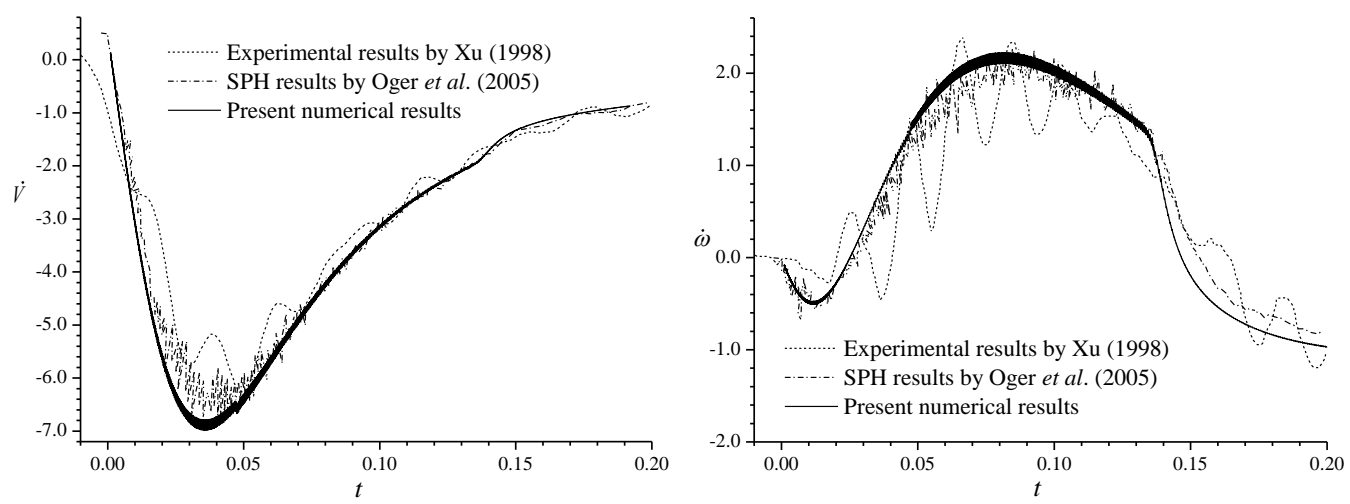

Fig. 6 Results for water entry of an asymmetric wedge with $\gamma_{1}=25^{\circ}, \gamma_{2}=15^{\circ}, m=0.1366, l=0.3541, I_{Z Z}=0.0026$, $F r=1.4142$. (a) vertical acceleration, and (b) rotational acceleration.

\subsection{Case studies}

The centre of gravity of the wedge in Fig.1, where the rotational centre is located, is above its top, based on the assumption that there may be a structure attached to the wedge in practical problems. Some data of the wedge are given in Table. 1, which are used in the subsequent simulations unless specified specifically. This wedge model was also used in Wu et al. [10], in which the wedge vertically entering water in freefall was investigated numerically and experimentally. Then Xu et al. [12] used this wedge to study oblique water entry in free fall within three degrees of freedom. However the wedge in their simulations had infinite length and there was no flow detachment, and the gravity was also ignored.

\begin{tabular}{c|c|c|c}
\hline $\begin{array}{c}\text { Half inner } \\
\text { angle: } \gamma\end{array}$ & Mass: $m$ & $\begin{array}{c}\text { Distance between the tip of the } \\
\text { wedge to the mass center: } l\end{array}$ & Inertia: $I_{Z Z}$ \\
\hline $45^{\circ}$ & 2.5 & 1.25 & 28.125 \\
\hline
\end{tabular}

Tab. 1 The data of the wedge.

\subsubsection{Vertical entry of a symmetrical wedge}

We consider the wedge with initial heel angle $\theta_{0}=0^{\circ}$ entering vertically into water at speed of $F r=3$. Figs. $7 \mathrm{a}$ and $7 \mathrm{~b}$ show respectively the variation of acceleration and velocity with $s$. The initial acceleration is that due to gravity and is equal to $1 / F r^{2}$, and the initial velocity is 1 , based on nondimensionalisation. The acceleration is positive (downwards) for very short period of time, within which the vertical velocity increases. Then the acceleration changes the sign, and the velocity starts decreasing. The acceleration varies rapidly initially because of the large impact force due to larger velocity and rapid increase of the wetted surface. When the entry velocity slows down because of the action of the hydrodynamic force and when the wetted surface no longer changes after flow detachment, impact force decreases and therefore the acceleration. 

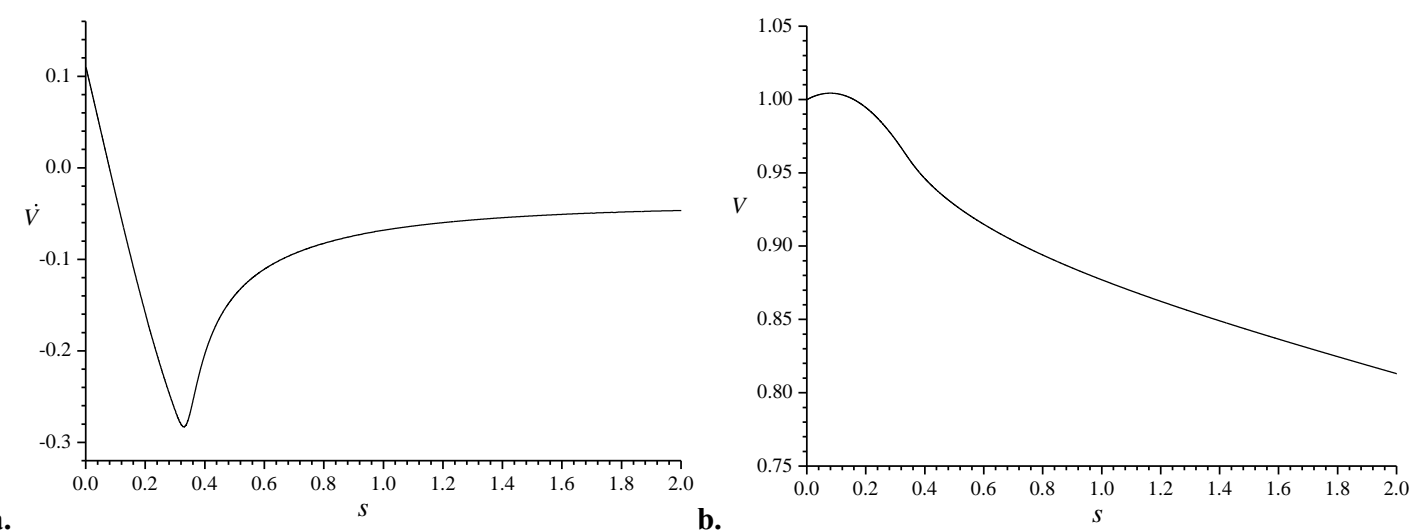

Fig. 7 Vertical entry of a symmetrical wedge with $F r=3, \theta_{0}=0^{\circ}$. (a) vertical acceleration, (b) vertical velocity.

Fig. $8 \mathrm{a}$ and $8 \mathrm{~b}$ give the free surface profiles and pressure distributions at different $s . L$ is the length of the body surface, and $d$ is the length coordinate measured from the tip of the wedge to a node on the wedge surface. $d / L$ varies from -1 to 0 on the left-hand side of the wedge, and from 0 to 1 on the right-hand side. When $s=0.05$ and $s=0.15$, or at the earlier stage during impact, a thin jet is fully attached to the body surface. Since the velocity has decreased, the pressure distribution at $s=0.15$ is lower than that at $s=0.05$. When $s=0.25$, the jet has detached from the knuckle but its root is still attached to the body surface. When $s=0.40$, the jet root has departed from the body surface, and the pressure distribution changes significantly. The larger pressure gradient near the jet root before the detachment has disappeared. The pressure variation along the body surface becomes much milder.

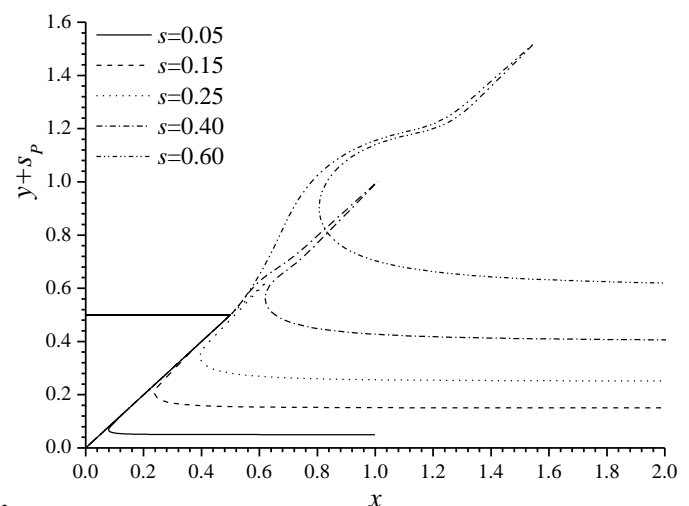

a.

Fig. 8 Vertical entry of a symmetrical wedge with $F r=3, \theta_{0}=0^{\circ}$. (a) free surface profile, (b) pressure distribution. and $9 \mathrm{~b}$ show the acceleration and velocity of wedges with $\gamma=30^{\circ}, 45^{\circ}$ and $60^{\circ}$. A wedge with larger $\gamma$ or a smaller deadrise angle will have much larger impact force upwards. It leads to a much larger upwards acceleration. Because of that, the speed of the wedge decreases more quickly, as shown in Fig.9b, which in turn reduces the impact force. Therefore Fig.9a also shows within certain $s$, the acceleration of the wedge with $\gamma=60^{\circ}$ is smaller than that of the wedge with $\gamma=45^{\circ}$. As $s$ increases, the hydrodynamic force acting on a wedge gradually balances its weight and the acceleration approaches zero. 

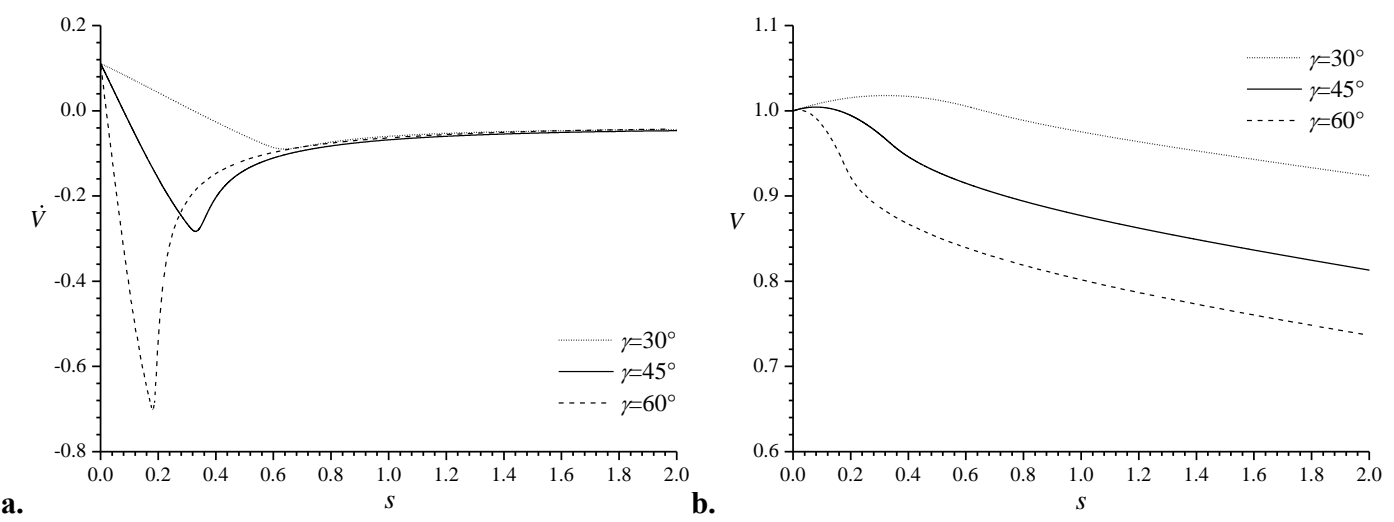

Fig. 9 Vertical entry of symmetrical wedges with different $\gamma(F r=3)$. (a) vertical acceleration, (b) vertical velocity.

Figs. 10 and 11 give the free surface profiles and pressure distributions at different $s$. When $s=0.1$, the thin jet flow is still attached on the body surface. The wedge with larger $\gamma$ has a much longer jet, and the flow will detach from the knuckle earlier, as shown in Fig. 10a. In Fig. 10b, a wedge with larger $\gamma$ has larger pressure, and larger pressure gradient. Fig. 10b can well explain that the wedge with larger $\gamma$ has larger upward acceleration. When $s=0.7$, the jet roots for all $\gamma$ have detached from the corresponding knuckle. The free surface profiles in Fig.11a have changed significantly, and they differ greatly from each other. In Fig. $11 \mathrm{~b}$, the large pressure gradient for $\gamma=60^{\circ}$ before flow detachment has disappeared. We have seen from Fig. 9a that the vertical hydrodynamic force gradually balances the weight. Since the weights of the wedges at different $\gamma$ are equal, and the vertical force acting on the wedge can be obtained from the pressure integration over its wetted surface $S_{0}$, the pressure distributions for different $\gamma$ have a very small difference, although the vertical velocities of those wedges are different (Fig. 9b).
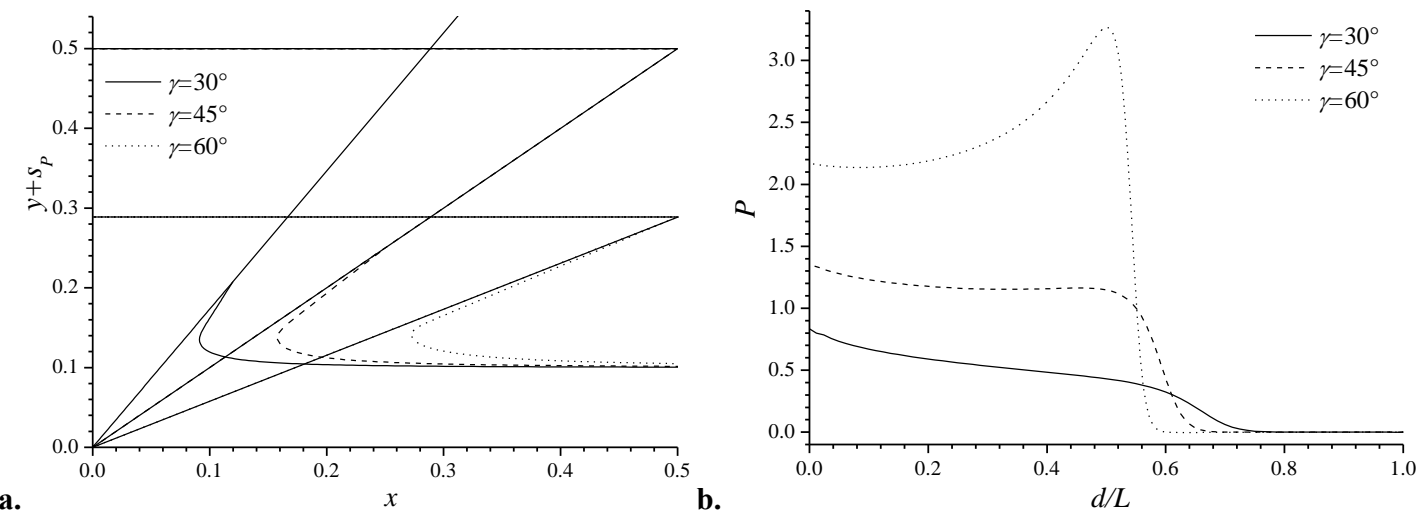

Fig. 10 Vertical entry of symmetrical wedges with different $\gamma(F r=3)$ at $s=0.1$. (a) free surface profile, (b) pressure distribution. 

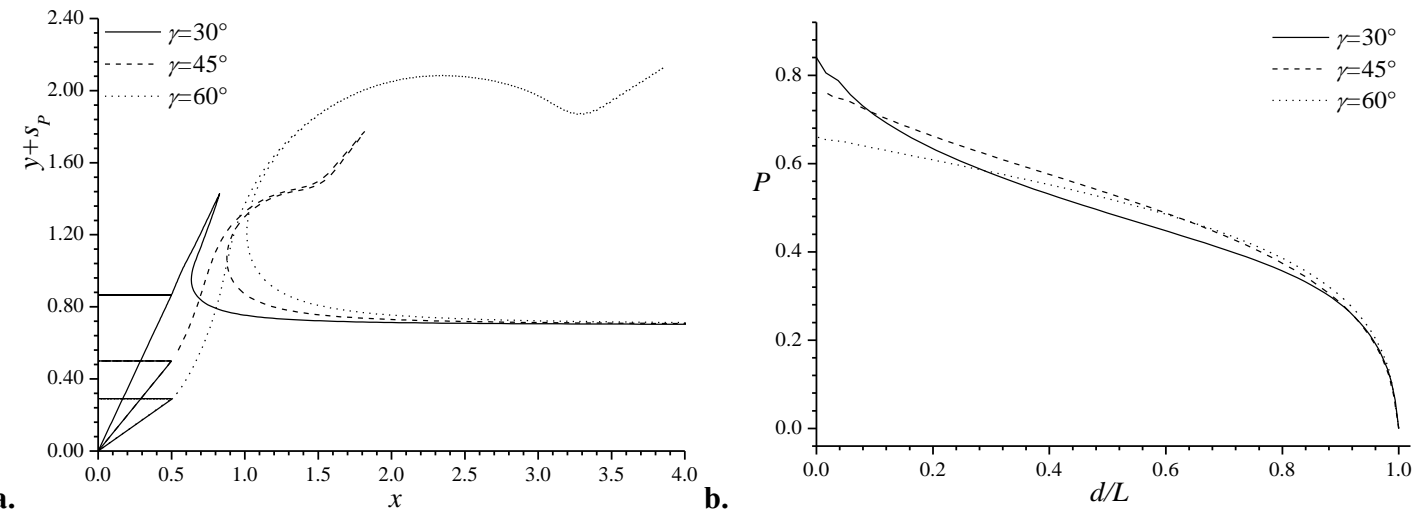

Fig. 11 Vertical entry of symmetrical wedges with different $\gamma(F r=3)$ at $s=0.7$. (a) free surface profile,

(b) pressure distribution.

\subsubsection{Vertical entry of an asymmetrical wedge}

We now consider water entry of a wedge which is asymmetric about $y$-axis and $F r=3$. The initial horizontal and rotational velocities are zero. Fig. 12 shows the acceleration and velocity components in three modes for the wedges with initial heel angle $\theta_{0}=0^{\circ}, 5^{\circ}, 10^{\circ}$ and $15^{\circ}$, respectively. The influence of $\theta_{0}$ on the horizontal and rotational accelerations and velocities is far more evident than that on the vertical ones. When $\theta_{0}$ is positive, the right deadrise angle is smaller than the left. As discussed previously, the body surface with smaller deadrise angle will lead to higher pressure. Subsequently, a negative horizontal force is developed. Furthermore, the imbalance of the forces on the both sides of the wedge creates a clockwise moment when the rotational centre is above the wedge. When $\theta_{0}$ increases the difference between the pressures on the right and left hand sides increases. It leads to a larger negative horizontal acceleration, as well as a larger clockwise rotational acceleration, as shown in Fig. 12a and 12c, respectively. Since the initial horizontal and rotational velocities are zero, the horizontal and rotational accelerations lead to an increasing horizontal velocity and an increasing clockwise rotational velocity. Form Eqs. (32) and (33), the rotational centre will keep moving towards left hand side and the heel angle will keep increasing. When the jet root detaches from the knuckle, the pressure on the right hand side reduce significantly. Subsequently, the negative horizontal force decreases, as well as the clockwise moment. Those can be reflected by the acceleration in Fig.12a and 12c.
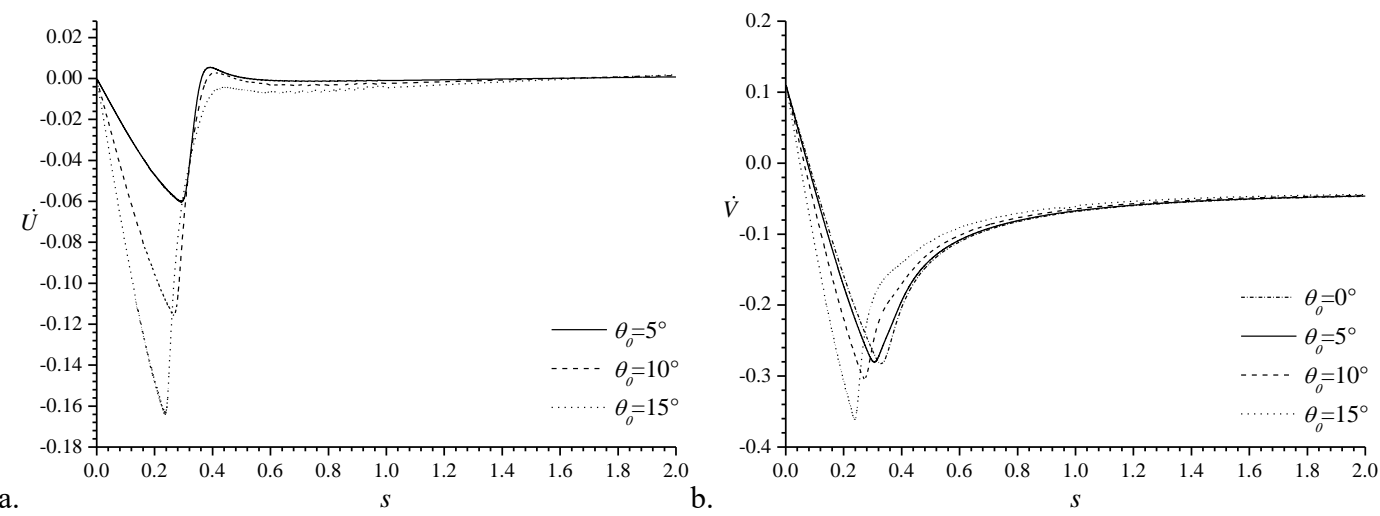

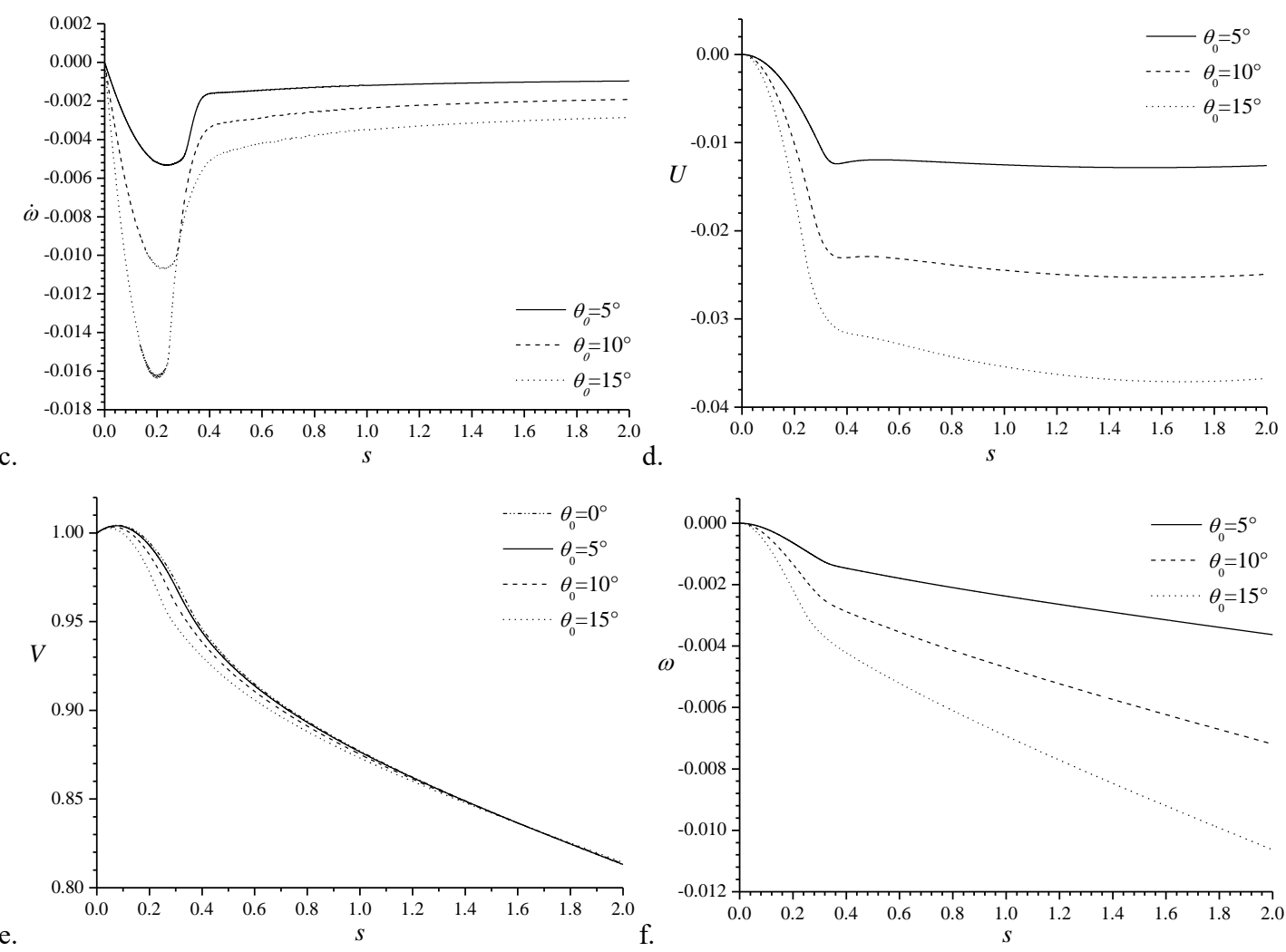

Fig. 12 Vertical entry of asymmetrical wedges with different $\theta_{0}(F r=3)$. (a) horizontal acceleration, (b) vertical acceleration, (c) rotational acceleration, (d)horizontal velocity, (e)vertical velocity, (f) rotational velocity.
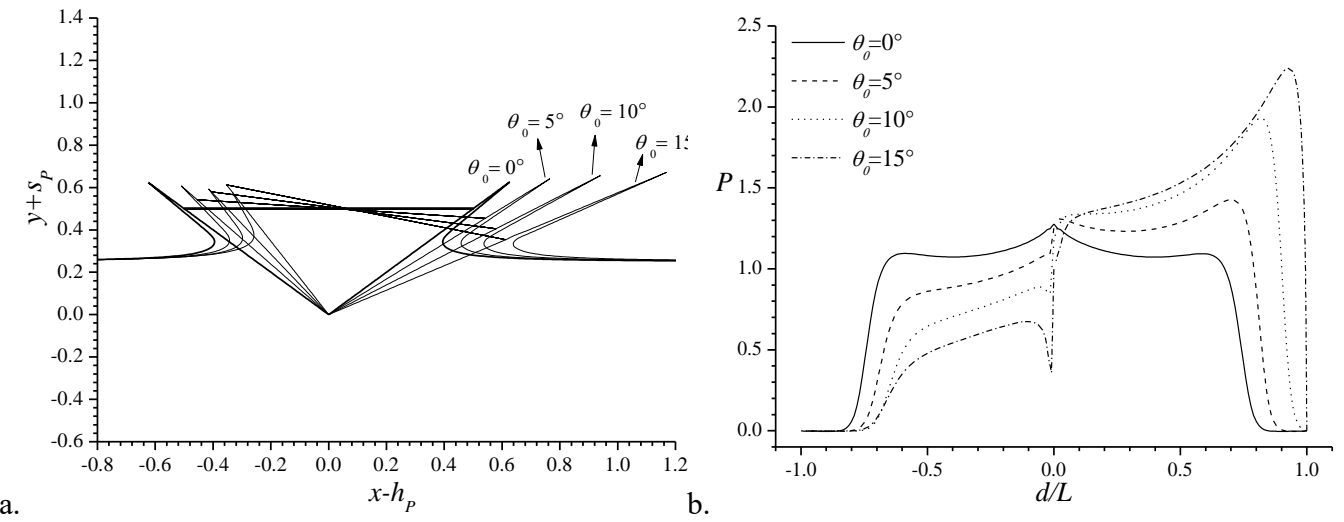

Fig. 13 Vertical entry of asymmetrical wedges with different $\theta_{0}(F r=3)$ at $s=0.25$. (a) free surface profile,

(b) pressure distribution.
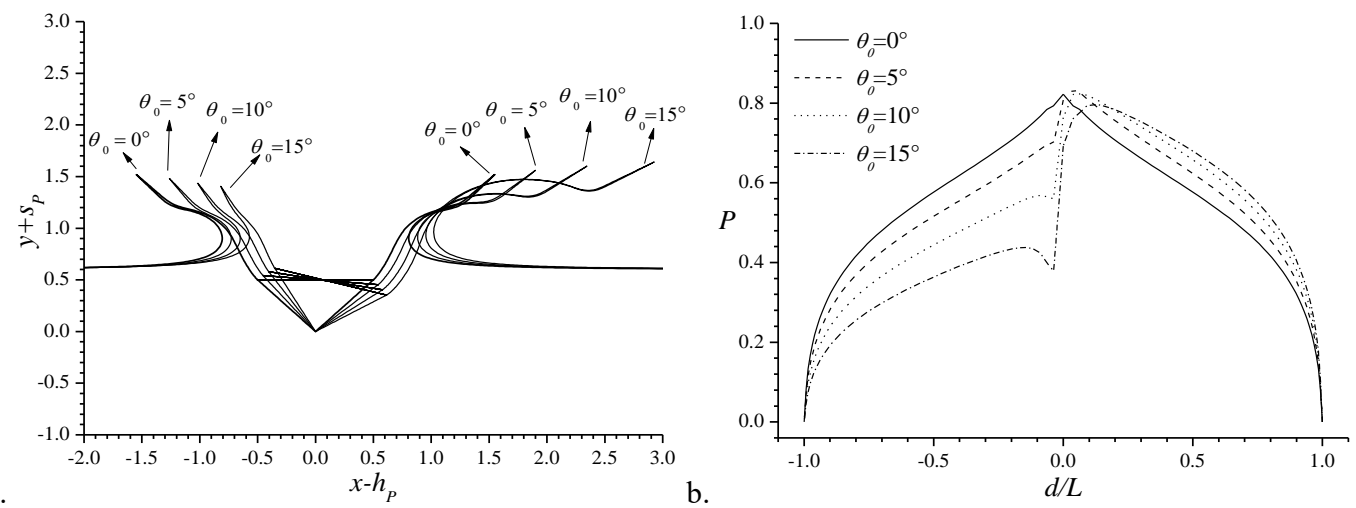

Fig. 14 Vertical entry of asymmetrical wedges with different $\theta_{0}(F r=3)$ at $s=0.65$. (a) free surface profile, 
(b) pressure distribution.

Fig. 13 and Fig. 14 show the results at $s=0.25$ and $s=0.65$, respectively. Consistent with Fig. 12, these two figures show continuous clockwise rotating of the wedge, and $\gamma_{1}$ and $\gamma_{2}$ keep increasing and decreasing, respectively. This is obviously due to the fact that the centre of the gravity is above the wedge, and the pressure force on the body provides a clockwise moment when $\theta>0$. It is clearly observed that the pressure near the tip of the wedge changes rapidly. In the experiment carried out by Judge et al. [21], air pocket near the tip of the wedge has been observed, which means the water has departed from the tip of the wedge. An appropriate approach to account for such a phenomenon is to use the coupled hydrodynamic and aerodynamic model in the time domain. However, it is beyond the scope of the current work. At the tip of the body, the flow is assumed to be always attached in the present paper.

We now consider an example with the rotating centre at the tip of the wedge, similar to that of an arrow. Results similar to those Fig.12a-f are given in Figs. $15 \mathrm{a}-\mathrm{f}$ for $\theta_{0}=10^{\circ}$ and $l=0$. The difference in the horizontal and vertical accelerations and velocities is small, but that for rotational ones is very obvious. This is because the magnitudes of the rotational acceleration and velocity are smaller than the horizontal and vertical ones. At the early stage, the change of the deadrise angle due to small rotational velocity is small, and subsequently the change of the pressure distribution on the body surface is also small. However, as $s$ increases, the difference between the results in the two cases becomes more evident, as shown in Figs. 15a-b and 15d-e. Since $l$ is different, the rotational moments due to the imbalance of the forces acting on the left and right body surface are in opposite directions. The rotational velocity for $l=0$ in Fig. $15 \mathrm{f}$ is anticlockwise, and subsequently the heel angle will decrease based on Eq. (32)
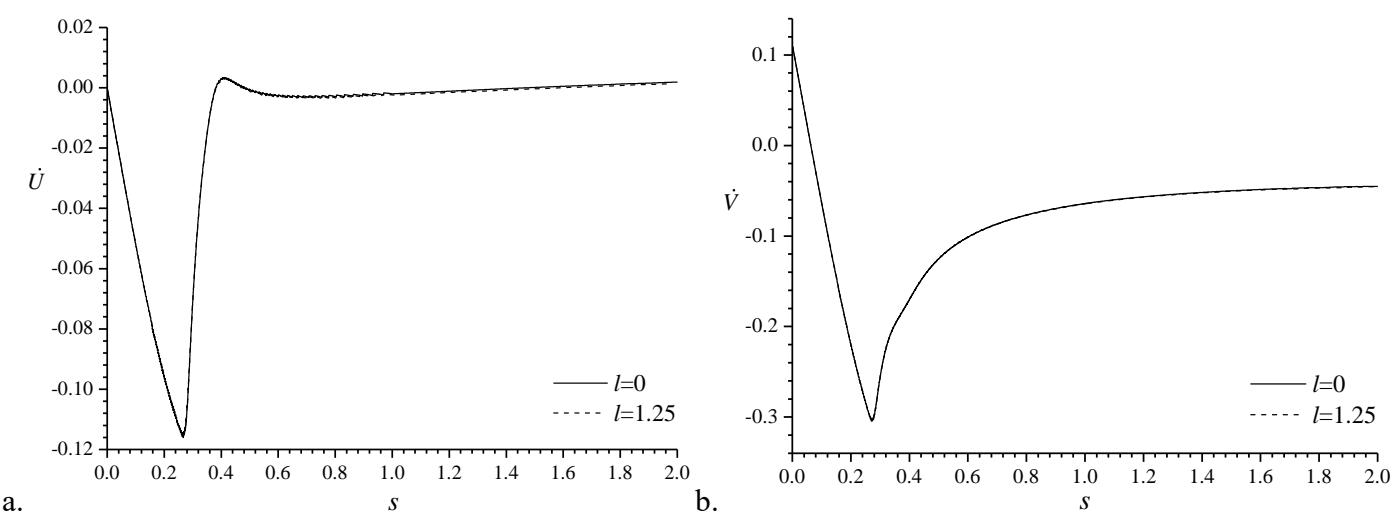

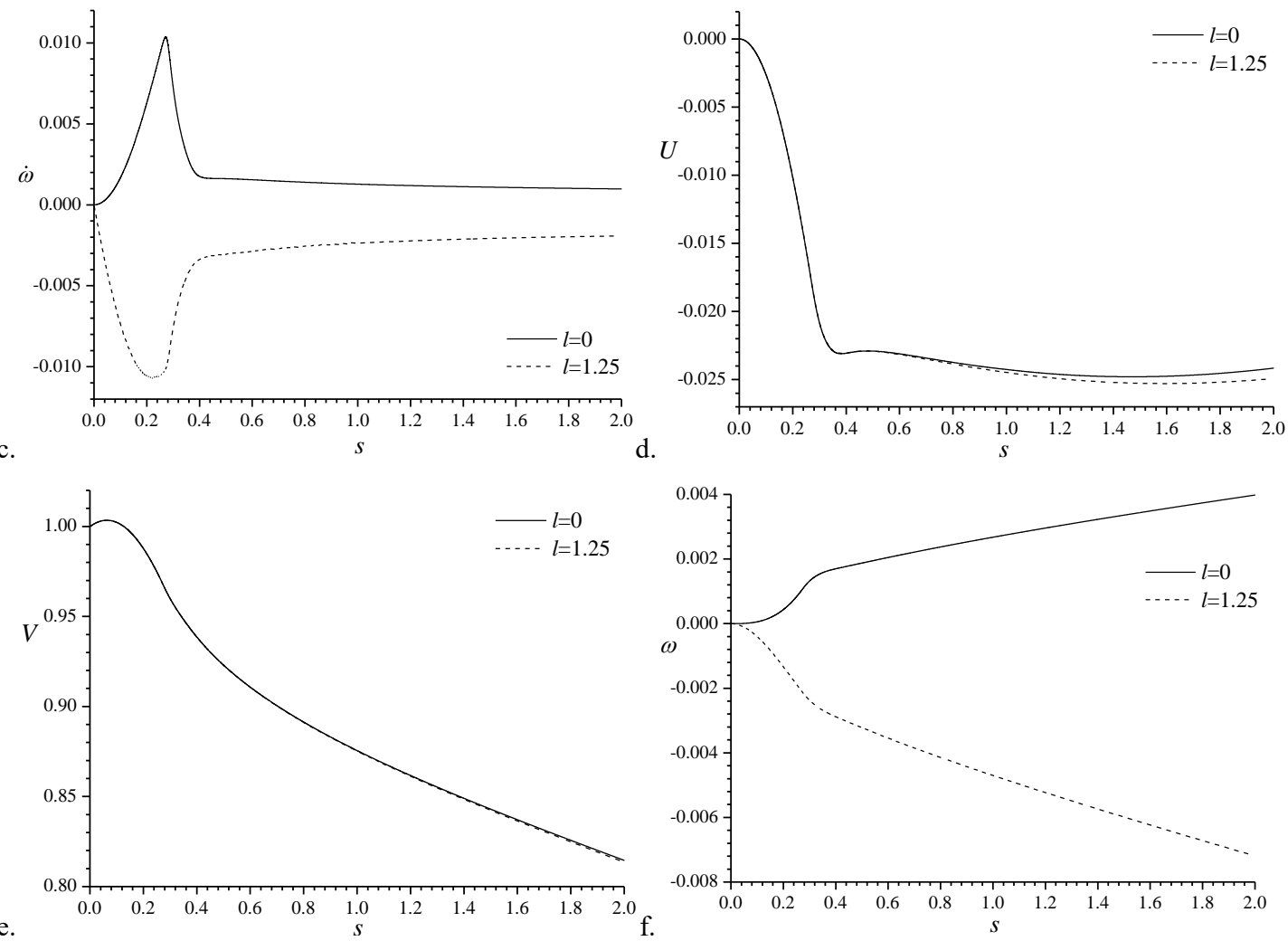

Fig. 15 Comparison of vertical entry of asymmetrical wedges $\left(\theta_{0}=10^{\circ}, F r=3\right)$ with different $l(F r=3)$. (a) horizontal acceleration, (b) vertical acceleration, (c) rotational acceleration, (d)horizontal velocity, (e)vertical velocity, (f) rotational velocity.

Wang et al. [22] analysed vertical water entry in freefall of an infinite wedge. The mass of a wedge was found to have a significant effect on the hydrodynamic load and the motion of the wedge. We now consider the asymmetric wedges with $\theta_{0}=10^{\circ}$ of different mass $m$ entering water, while other parameters remain the same as those in Fig.12. Fig. 16 shows the acceleration and velocity components in three modes for the wedges with $m=1.25,2.50$ and 7.50 , respectively. The impact loading will depend only the body velocity and acceleration. Thus at the initial stage, the forces on all the wedges will be similar. This leads to that a lighter wedge will have to a larger translational acceleration. As the rotational inertial is the same for the three wedges, all their angular accelerations are very close for a much longer period of time. However, as translational velocity decreases faster for a lighter wedge, the loading will also be smaller. This subsequently leads to a smaller angular acceleration. 

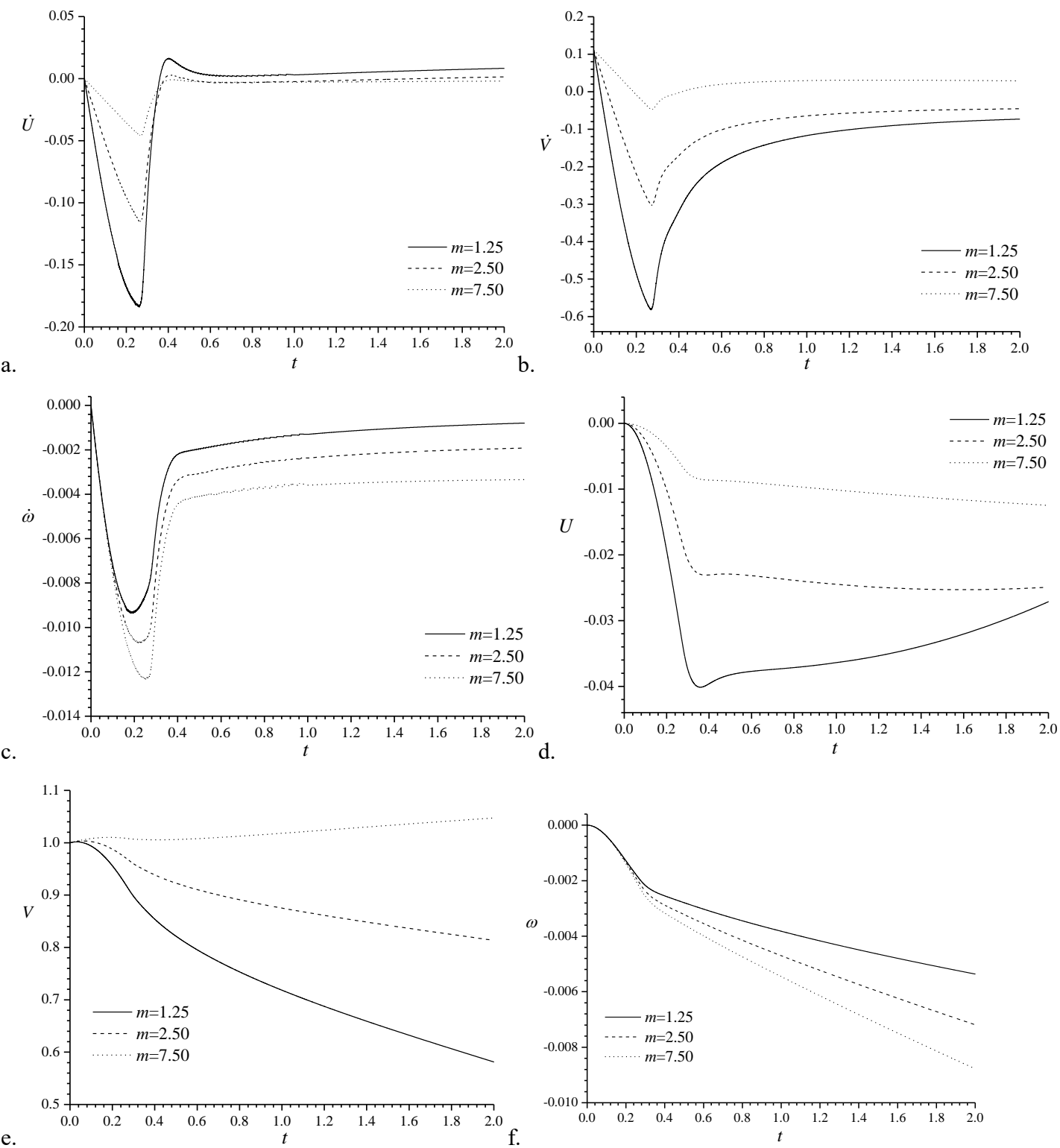

Fig. 16 Comparison of vertical entry of asymmetrical wedges $\left(\theta_{0}=10^{\circ}, F r=3\right)$ with different $m$. (a) horizontal acceleration, (b) vertical acceleration, (c) rotational acceleration, (d) horizontal velocity, (e)vertical velocity, (f) rotational velocity.

\subsubsection{Oblique entry of an asymmetrical wedge}

We now consider oblique water entry of an asymmetric wedge with heel angle $\theta_{0}=10^{\circ}$ and $F r=3$, and with different horizontal velocities $U_{0}=-0.3,0,0.3$ and 0.5 . The initial rotational velocity is taken as zero. Fig. 17 shows the acceleration and velocity components in three modes. When $U_{0}$ is positive, it enhances the negative horizontal force. Larger $U_{0}$ leads to a larger deceleration in horizontal direction. Large $U_{0}$ also leads to large acceleration in vertical direction and larger magnitude of rotational acceleration, which is very much due to the increase of pressure on the right side of the wedge, as shown in Fig.18 and Fig. 19. When $U_{0}<0$, it cancels part of the horizontal force due to $\theta_{0}$. Fig.17a shows that the horizontal acceleration component is very small for a period of time. 

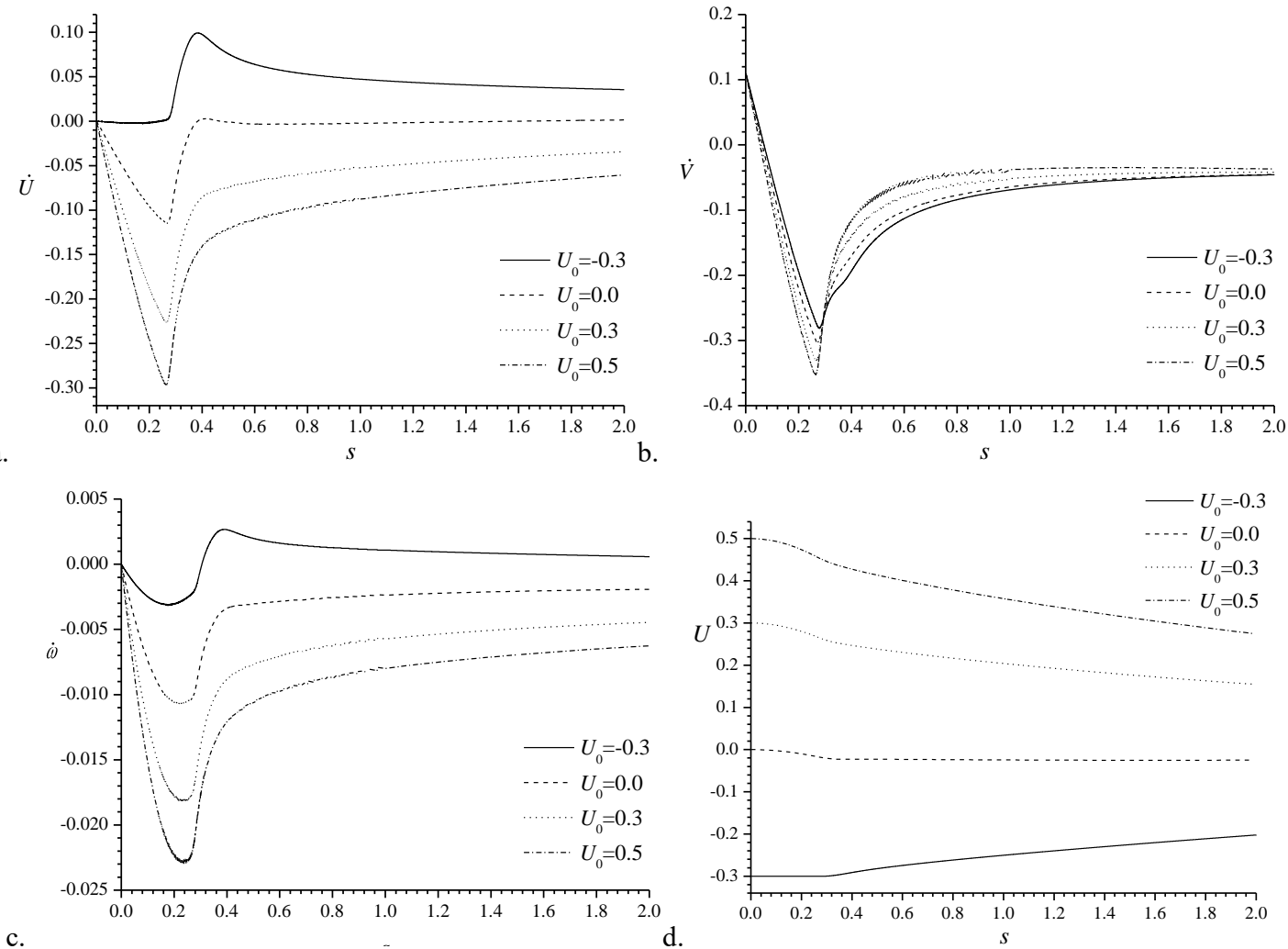

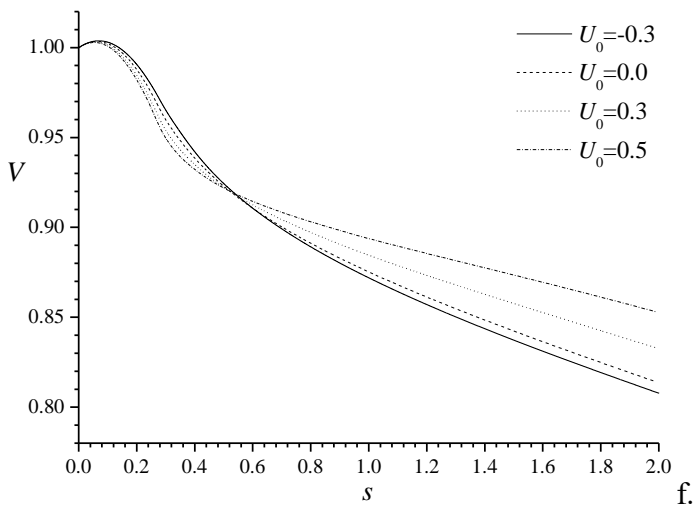

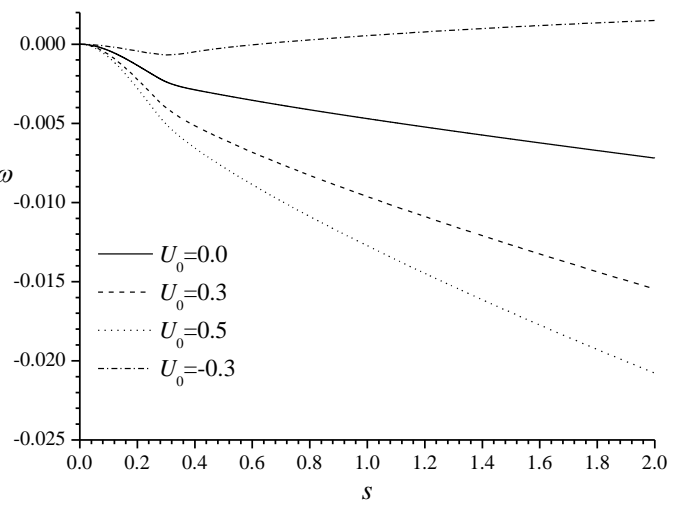

Fig. 17 Oblique entry of asymmetrical wedges with different $U_{0}\left(F r=3, \theta_{0}=10^{\circ}\right)$. (a) horizontal acceleration, (b) vertical acceleration, (c) rotational acceleration, (d)horizontal velocity, (e)vertical velocity, (f) rotational velocity.
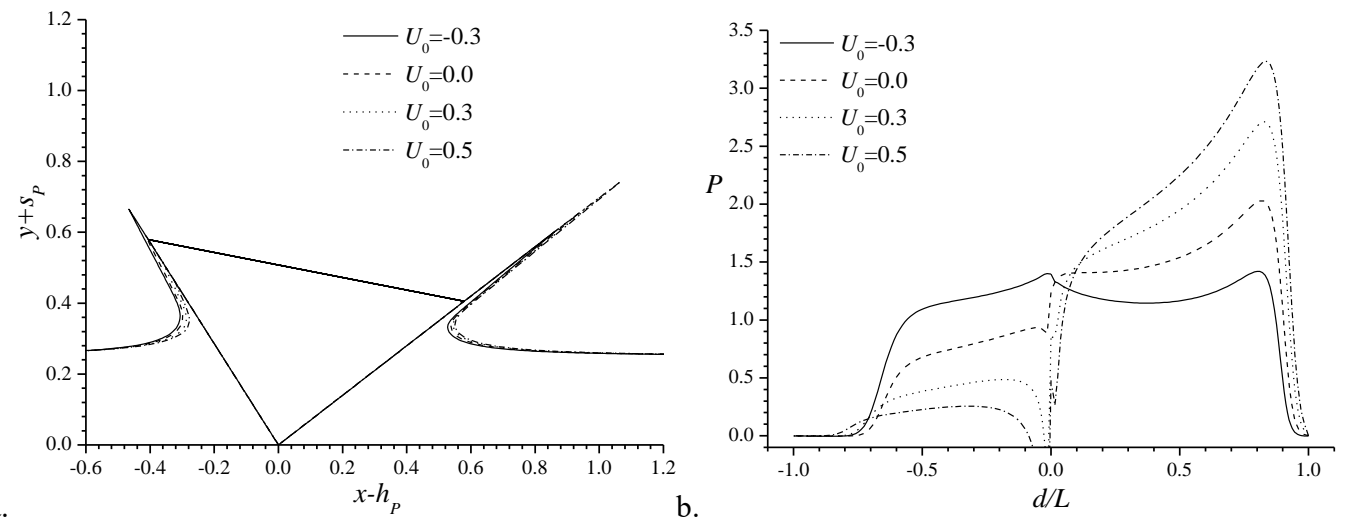

Fig. 18 Oblique entry of asymmetrical wedges with different $U_{0}\left(F r=3, \theta_{0}=10^{\circ}\right)$ at $s=0.25$. (a) free surface profile, (b) pressure distribution. 

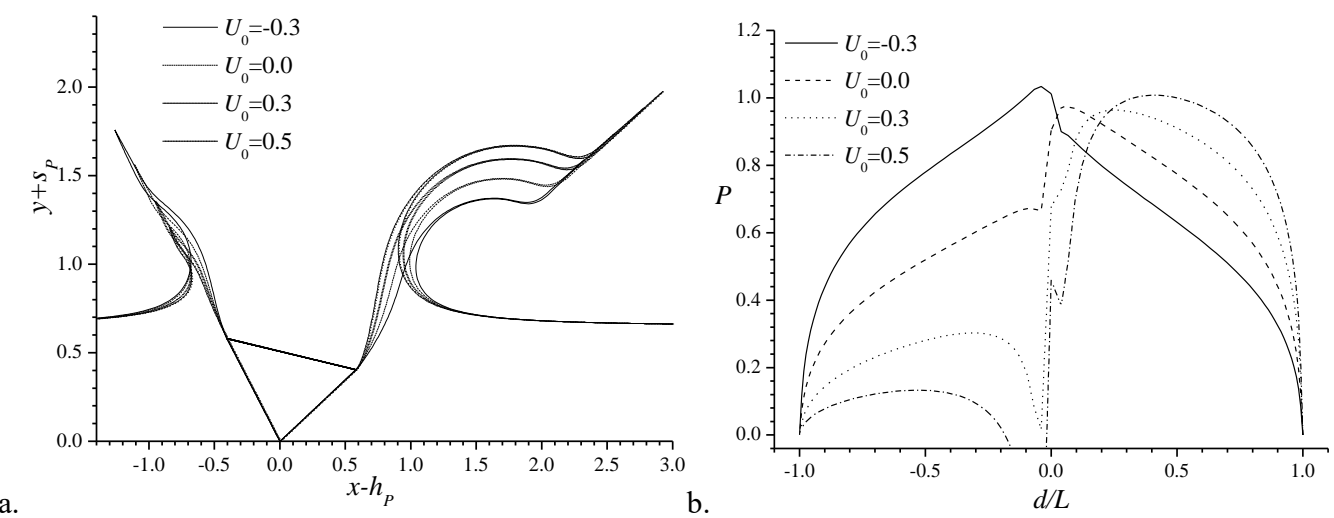

Fig. 19 Oblique entry of asymmetrical wedges with different $U_{0}\left(F r=3, \theta_{0}=10^{\circ}\right)$ at $s=0.65$. (a) free surface profile, (b) pressure distribution.

\subsubsection{Oblique entry of an asymmetrical wedge with initial rotational velocity}

We finally consider oblique water entry of an asymmetric wedge with heel angle $\theta_{0}=10^{\circ}, F r=3$ and $U_{0}=0.3$, and with different rotational velocities $\omega_{0}=-0.1,0,0.1$ and 0.2 . Fig.20 shows the acceleration and velocity components in three modes, and Figs. 21 and 22 show the free surface profiles and pressure distributions at $s=0.2$ and $s=1.5$, respectively. When $\omega$ is positive, the rotational velocity is anticlockwise. In such a case the pressure on the right body surface increases, and that on the left hand body surface decrease. At earlier stage of $s=0.2$, the results at different $\omega_{0}$ are very close. This is mainly due to the fact that the entry speed is much larger than the rotational velocity and the effect of the translational velocity is dominant. At $s=1.5$ shown in Fig.22, the position of the wedge at different $\omega_{0}$ becomes very different, due to accumulation of rotation. As a result, the pressure distributions also become very different.
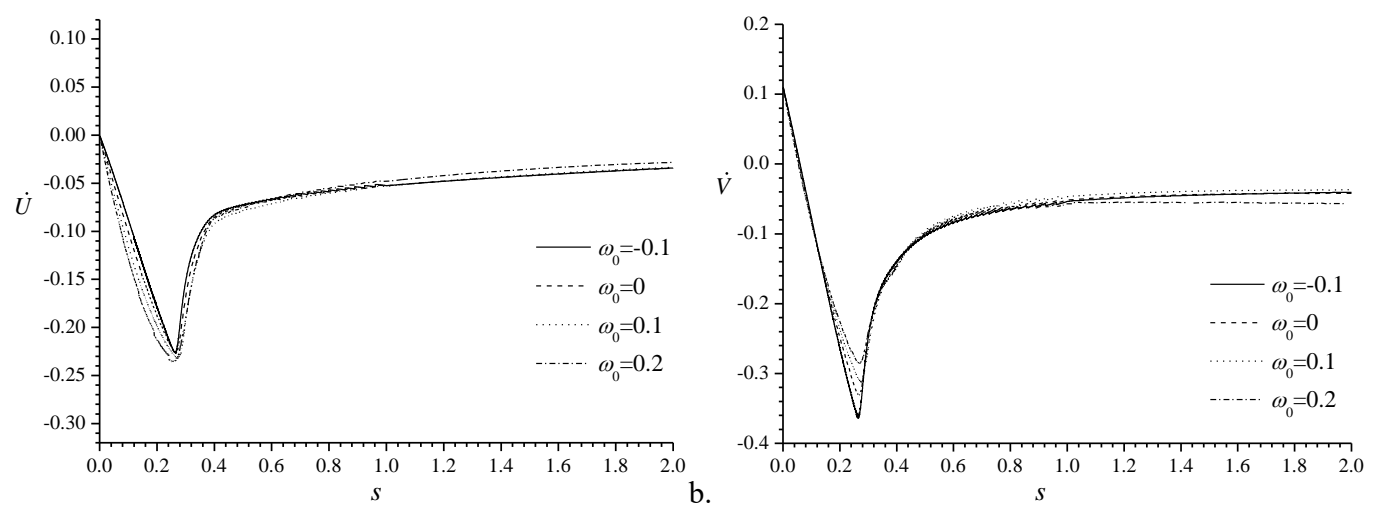

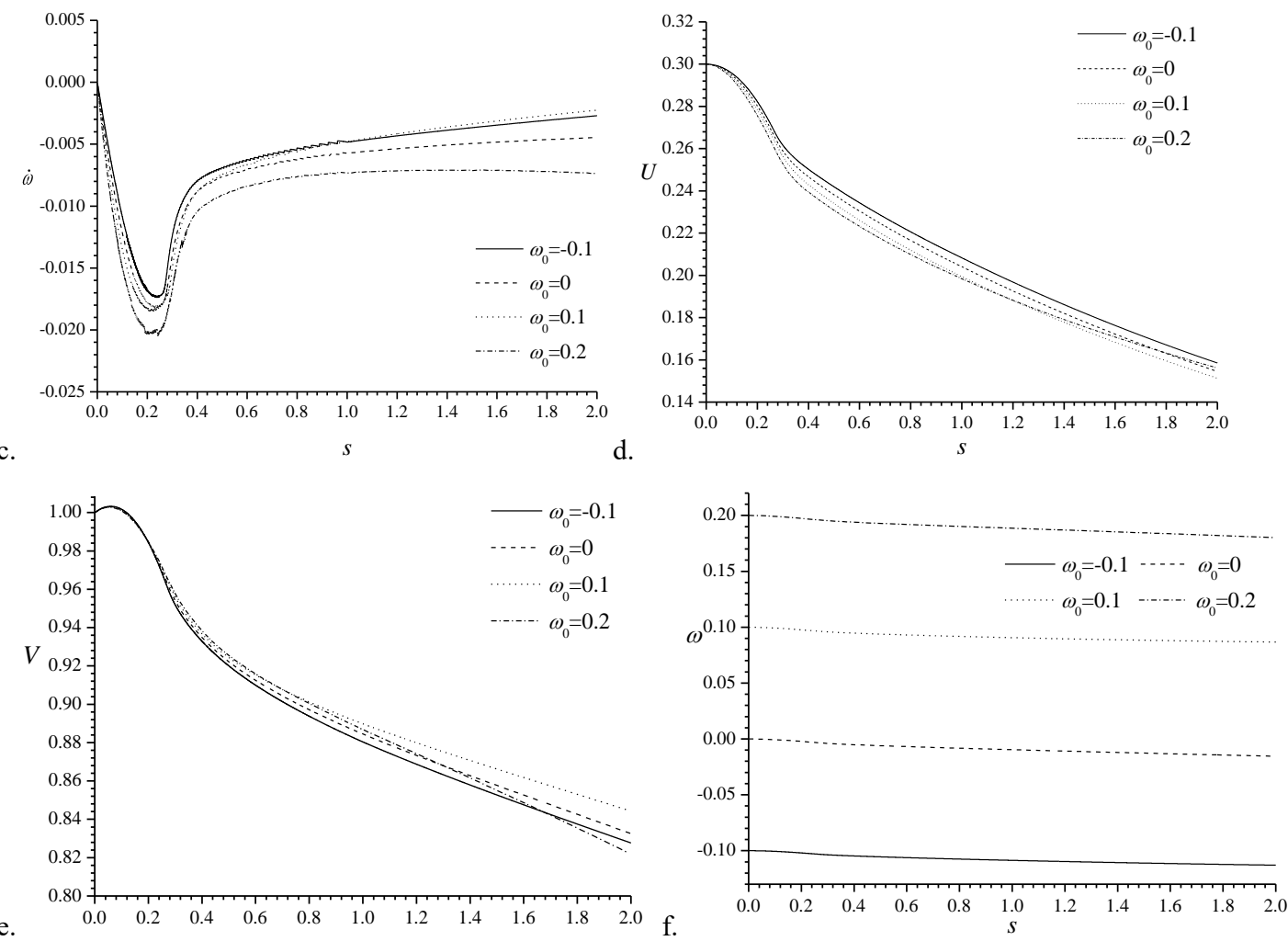

Fig. 20 Oblique entry of an asymmetrical wedge with different $\omega_{0}\left(F r=3, \theta_{0}=10^{\circ}, U_{0}=-0.3\right)$. (a) horizontal acceleration, (b) vertical acceleration, (c) rotational acceleration, (d)horizontal velocity, (e)vertical velocity, (f) rotational velocity.
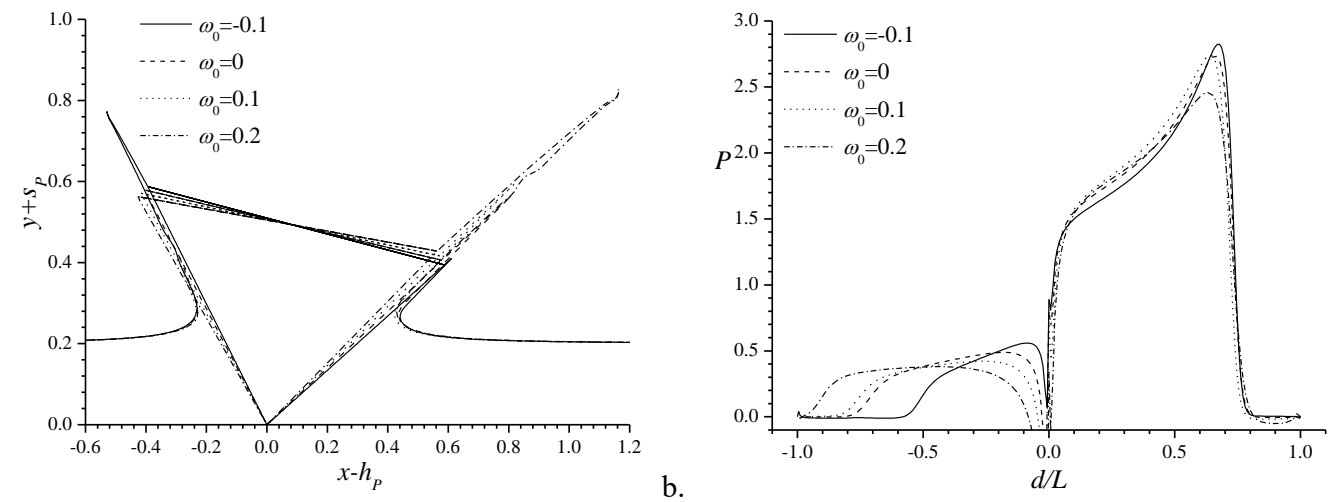

Fig. 21 Oblique entry of an asymmetrical wedge with different $\omega_{0}\left(F r=3, \theta_{0}=10^{\circ}, U_{0}=0.3\right)$ at $s=0.2$. (a) free surface profile, (b) pressure distribution.
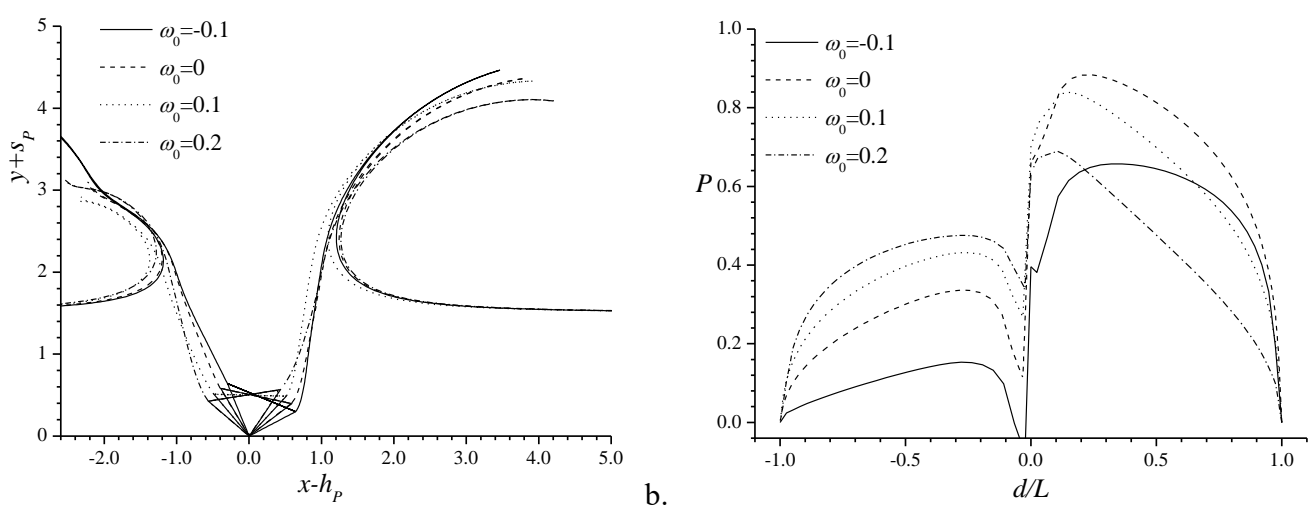
Fig. 22 Oblique entry of an asymmetrical wedge with different $\omega_{0}\left(F r=3, \theta_{0}=10^{\circ}, U_{0}=0.3\right)$ at $s=1.5$.

(a) free surface profile, (b) pressure distribution.

\section{Conclusions}

The problem of a finite asymmetric wedge obliquely entering water in free fall in three degrees of freedom is solved by using the boundary element method for the velocity potential. Flow detachment from the knuckle is also simulated. The thin free jet after flow detachment is treated using the momentum equation. The auxiliary function method is adopted to decouple the nonlinear mutual dependence between the body motion and the fluid flow. Through the obtained results, we can draw the following conclusions.

(1) When a body enters water vertically, there is a sharp reduction of downwards acceleration due to the large impact force. In fact the acceleration rapidly changes its direction and its magnitude can be much larger than acceleration due to gravity. As a result, the entry speed is reduced sharply. The impact force will decrease with the reduction of the falling speed of the body, especially when the wetted surface of the body no longer increases after flow detachment.

(2) For oblique entry of an asymmetrical body, the fluid force will set the body into motion of three degrees of freedom. These motions are fully coupled or the motion on one mode will be very much affected by the other two modes. This leads to a complex pattern of the body motion.

(3) The location of the centre of the body mass has crucial effect on the motion. When the centre above the wedge, the initial heel angle will lead to further increase of the heel angle. The body will potentially become unstable during water entry. When the centre is at the tip, the heel angle may decrease during water entry and the body motion is more likely to be stable. This is expected to be true when the centre is at the front part of the body.

(4) A small rotational velocity may not have significant effect on the results initially. However, due to accumulation of rotation, at later stage, difference between results at differential initial rotational velocities becomes evident.

\section{Acknowledgements}

This work is supported by Lloyd's Register Foundation through the joint centre involving University College London, Shanghai Jiaotong University and Harbin Engineering University, to which the authors are most grateful. Lloyd's Register Foundation helps to protect life and property by supporting engineering-related education, public engagement and the application of research.

This work is also supported by the National Natural Science Foundation of China (Grants number 11472088)

\section{References}

[1] Dobrovol'skaya, Z. N. Some problems of similarity flow of fluid with a free surface. J. Fluid Mech. 36 (1969) 805-829.

[2] Zhao, R., Faltinsen, O. M. Water entry of two-dimensional bodies. J. Fluid Mech. 246 (1993) 
$593-612$.

[3] Semenov, Y. A., Iafrati, A. On the nonlinear water entry problem of asymmetric wedges. Journal of Fluid Mechanics, 547 (2006) 231-256.

[4] Xu, G. D., Duan, W. Y., Wu, G. X. Numerical simulation of oblique water entry of an asymmetrical wedge. Ocean Engineering, 35(16) (2008) 1597-1603.

[5] Zhao, R., Faltinsen, O., \& Aarsnes, J. Water entry of arbitrary two-dimensional sections with and without flow separation. In: Proceedings of the 21 st symposium on naval hydrodynamics, 408423. Trondheim, Norway, National Academy Press, Washington, DC, USA., 1996

[6] Iafrati, A., Battistin, D. Hydrodynamics of water entry in presence of flow separation from chines. In 8th International Conference on Numerical Ship Hydrodynamics., 2003

[7] Tassin, A., Korobkin, A. A., Cooker, M. J. On analytical models of vertical water entry of a symmetric body with separation and cavity initiation. Applied Ocean Research, 48 (2014) 33-41.

[8] Bao, C. M., Wu, G. X., Xu, G. D. Simulation of water entry of a two-dimension finite wedge with flow detachment. Journal of Fluids and Structures, 65 (2016) 44-59.

[9] Semenov, Y. A., \& Wu, G. X. Water entry of an expanding wedge/plate with flow detachment. Journal of Fluid Mechanics, 797 (2016) 322-344.

[10] Wu, G. X., Sun H., He Y.S. Numerical simulation and experimental study of water entry of a wedge in free fall motion. Journal of Fluids and Structures 19 (2004) 277-289.

[11] Wu, G. X., Eatock Taylor, R. The coupled finite element and boundary element analysis of nonlinear interactions between waves and bodies. Ocean Engineering, 30(3) (2003) 387-400.

[12] Xu, G. D., Duan, W. Y., Wu, G. X. Simulation of water entry of a wedge through free fall in three degrees of freedom. In: Proceedings of the Royal Society of London Series A, 466 (2010) 2219-2239.

[13] Sun, H. A boundary element method applied to strongly nonlinear wave-body interaction problems. PhD thesis, Norwegian University of Science and Technology, 2007.

[14] Aarsnes, J. V. Drop test with ship sections-effect of roll angle, Report 603834.00. 01. Norwegian Marine Technology Research Institute, Trondheim, Norway, 1996.

[15] Wang, J., Lugni, C., Faltinsen, O. M. Experimental and numerical investigation of a freefall wedge vertically entering the water surface. Applied Ocean Research, 51 (2015) 181-203.

[16] Wu, G. X. Two-dimensional liquid column and liquid droplet impact on a solid wedge. The Quarterly Journal of Mechanics and Applied Mathematics, 60(4) (2007) 497-511.

[17] Lu C.H., He Y.S., Wu G.X. Coupled analysis of nonlinear interaction between fluid and structure during impact. Journal of Fluids and Structures 14 (2000) 127-146.

[18] $\mathrm{Wu}, \mathrm{G}$. X. Hydrodynamic force on a rigid body during impact with liquid. Journal of Fluids and Structures 12 (1998) 549-559.

[19] L. Xu, A.W. Troesch, R. Petterson, Asymmetric hydrodynamic impact and dynamic response of vessels, in: Proceedings of 17th International Conference on Offshore Mechanics and Arctic Engineering (1998) 98-320. 
[20] Oger, Guillaume, et al. Two-dimensional SPH simulations of wedge water entries. Journal of computational physics 213.2 (2006) 803-822.

[21] Judge, C., A. Troesch, and Marc Perlin. Initial water impact of a wedge at vertical and oblique angles. Journal of Engineering Mathematics 48.3-4 (2004) 279-303.

[22]Wang, Jingbo, Claudio Lugni, and Odd Magnus Faltinsen. Analysis of loads, motions and cavity dynamics during freefall wedges vertically entering the water surface. Applied Ocean Research 51 (2015) 38-53. 